\title{
Room-temperature electrical control of exciton flux in a van der Waals heterostructure
}

\author{
Dmitrii Unuchek ${ }^{1,2,4}$, Alberto Ciarrocchi ${ }^{1,2,4}$, Ahmet Avsar ${ }^{1,2}$, Kenji Watanabe ${ }^{3}$, Takashi Taniguchi ${ }^{3}$ \& Andras Kis ${ }^{1,2 *}$
}

Devices that rely on the manipulation of excitons-bound pairs of electrons and holes-hold great promise for realizing efficient interconnects between optical data transmission and electrical processing systems. Although exciton-based transistor actions have been demonstrated successfully in bulk semiconductorbased coupled quantum wells ${ }^{1-3}$, the low temperature required for their operation limits their practical application. The recent emergence of two-dimensional semiconductors with large exciton binding energies ${ }^{4,5}$ may lead to excitonic devices and circuits that operate at room temperature. Whereas individual two-dimensional materials have short exciton diffusion lengths, the spatial separation of electrons and holes in different layers in heterostructures could help to overcome this limitation and enable room-temperature operation of mesoscale devices ${ }^{6-8}$. Here we report excitonic devices made of $\mathrm{MoS}_{2}-\mathrm{WSe}_{2}$ van der Waals heterostructures encapsulated in hexagonal boron nitride that demonstrate electrically controlled transistor actions at room temperature. The long-lived nature of the interlayer excitons in our device results in them diffusing over a distance of five micrometres. Within our device, we further demonstrate the ability to manipulate exciton dynamics by creating electrically reconfigurable confining and repulsive potentials for the exciton flux. Our results make a strong case for integrating two-dimensional materials in future excitonic devices to enable operation at room temperature.

Solid-state devices use particles and their quantum numbers for their operation, with electronics being the ubiquitous example. The need to improve power efficiency of charge-based devices and circuits is motivating research into new devices that would rely on other principles. Candidates so far include spintronics and photonics ${ }^{9,10}$. Excitons-electrically neutral quasi-particles formed by bound electrons and holes-can also be manipulated in solid-state systems. The development of such excitonic devices has so far been hindered by the absence of a suitable system that would enable room-temperature manipulation of excitons, limiting the expansion of the field. Here, we demonstrate room-temperature excitonic devices based on atomically thin semiconductors. These devices could open the way for wider studies and applications of excitonic devices in the academic and industrial sectors ${ }^{11}$. Many applications can be envisaged, because excitons could be used to efficiently couple optical data transmission and electronic processing systems. Although fast optical switches have already been

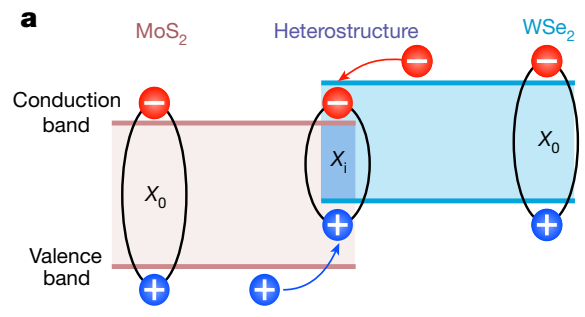

b
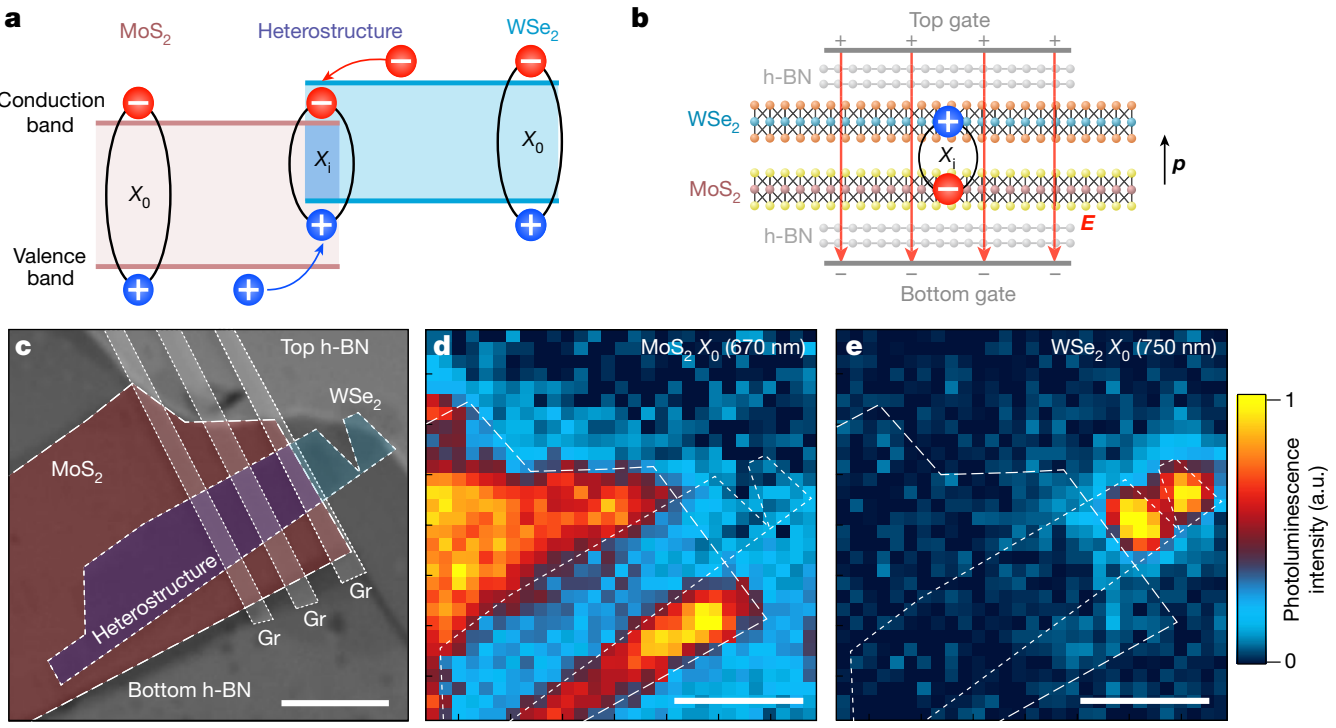

Fig. 1 Interlayer excitons in the $\mathrm{WSe}_{2}-\mathrm{MoS}_{2}$ van der Waals heterostructure. a, Type-II band alignment in the $\mathrm{WSe}_{2}-\mathrm{MoS}_{2}$ heterostructure with intralayer $\left(X_{0}\right)$ and interlayer $\left(X_{\mathrm{i}}\right)$ excitons. The red and blue areas represent the bands in the two materials and the heterobilayer. Positive and negative symbols indicate holes and electrons, respectively. $\mathbf{b}$, Schematic depiction of the $\mathrm{WSe}_{2}-\mathrm{MoS}_{2}$ heterostructure, showing the heterobilayer encapsulated in hexagonal boron nitride (h-BN) and the top and bottom gates. The interlayer exciton has a permanent out-of-plane dipole moment $\boldsymbol{p}$ that allows manipulation via the electric field $\boldsymbol{E}$. c, False-colour optical image of the device, highlighting the different materials. d, e, Spatial maps of photoluminescence at $670 \mathrm{~nm}$ (d) and $750 \mathrm{~nm}(\mathbf{e})$, corresponding to $\mathrm{MoS}_{2}$ and $\mathrm{WSe}_{2}$ intralayer excitonic resonances, respectively. Photoluminescence is quenched in the heterostructure area owing to efficient charge transfer. Scale bars, $5 \mu \mathrm{m}$. a.u., arbitrary units. 
demonstrated $^{12,13}$, the comparably large size (about $\left.10 \mu \mathrm{m}\right)^{14,15}$ of such devices limits packing density. This can be overcome in excitonic devices, the characteristic size of which is determined by that of electronic field-effect transistors (FETs).

Owing to their finite binding energy $E_{\mathrm{b}}$, excitons can exist up to temperatures of around $T \propto E_{\mathrm{b}} / k_{\mathrm{B}}$, where $k_{\mathrm{B}}$ is the Boltzmann constant. In a conventional III-V-semiconductor coupled quantum well with a size of a few nanometres, the relatively small binding energy of around $10 \mathrm{meV}$ permits the observation of excitons only at cryogenic temperatures (less than $100 \mathrm{~K})^{3}$. To reach higher temperatures, different materials are required. To this end, systems with higher $E_{\mathrm{b}}$ (in the range of tens of millielectronvolts) have been explored more recently, such as $(\mathrm{Al}, \mathrm{Ga}) \mathrm{N} /$ $\mathrm{GaN}$ (ref. ${ }^{16}$ ) or $\mathrm{ZnO}$ (ref. ${ }^{17}$ ). Two-dimensional semiconductors such as transition-metal dichalcogenides have even larger exciton binding energies, which can exceed $500 \mathrm{meV}$ in some cases owing to strong quantum confinement ${ }^{4,5}$. This could enable the realization of excitonic devices that operate at room temperature ${ }^{18}$.

Although intralayer excitons have relatively short lifetimes (about $10 \mathrm{ps})^{7,19}$, the spatial separation of holes and electrons in interlayer excitons results in lifetimes more than two orders of magnitude longer, well in the nanosecond range ${ }^{6}$. For the device presented here, we take advantage of interlayer excitons in an atomically thin $\mathrm{MoS}_{2}-\mathrm{WSe}_{2}$ heterostructure. Type-II band alignment ${ }^{20,21}$ (Fig. 1a) results in charge separation between the constituent materials, with electrons and holes residing in $\mathrm{MoS}_{2}$ and $\mathrm{WS} \mathrm{S}_{2}$, respectively. The formation of indirect excitons is marked by the appearance of a new photoluminescence emission peak ${ }^{22}$, redshifted by about $75 \mathrm{meV}$ with respect to the intralayer exciton of the $\mathrm{WSe}_{2}$ monolayer. In Extended Data Fig. 1b we present a typical photoluminescence spectrum obtained from such a heterostructure on $\mathrm{SiO}_{2}$, in which the spectral signature of the interlayer exciton is clearly visible (dark blue line), together with the individual $\mathrm{WSe}_{2}$ and $\mathrm{MoS}_{2}$ monolayers (blue and red lines, respectively). Recent reports ${ }^{23}$ suggest that excitons in the $\mathrm{MoS}_{2}-\mathrm{WSe}_{2}$ system are not only spatially indirect, but also momentum-indirect owing to lattice mismatch. The phonon-assisted nature of the emission process further reduces the exciton recombination rate, yielding a longer lifetime ${ }^{8,24}$. Such an extended lifetime can be used to obtain interlayer exciton diffusion over a scale of micrometres, even at room temperature.

To obtain a pristine surface, the heterostructure is encapsulated in hexagonal boron nitride and annealed in high vacuum. Multiple transparent top gates are fabricated out of few-layer graphene. This double-gate configuration allows us to apply a vertical electric field without changing the carrier concentration in the $\mathrm{MoS}_{2}-\mathrm{WSe}_{2}$ heterostructure. In Fig. 1c we show a false-colour optical micrograph of the resulting stack. We characterize the structure by using photoluminescence mapping at room temperature, under 647-nm excitation. In Fig. 1d, e and Extended Data Fig. 1 we show the intralayer emission distribution at the wavelengths characteristic of $\mathrm{MoS}_{2}(670 \mathrm{~nm}), \mathrm{WSe}_{2}$ $(760 \mathrm{~nm})$ and the interlayer exciton $(785 \mathrm{~nm})$. Whereas individual monolayers appear to be homogeneously bright, emission from the heterostructure region is uniformly quenched by more than three orders of magnitude, owing to the efficient charge transfer between layers $^{24}$. Even with this strong quenching, we are able to detect the interlayer peak in the photoluminescence spectra (Extended Data Fig. 2), confirming the generation of interlayer excitons. Because this effect has a central role in our work, we fabricated three more heterostructures encapsulated in hexagonal boron nitride, confirming the reproducibility of this result (Extended Data Fig. 3).

Given that excitons do not carry a net electric charge, we do not expect their flow to be influenced by the direct application of an in-plane electric field. However, the confinement of oppositely charged carriers in different layers results in a well-defined interlayer-exciton dipole moment $\boldsymbol{p}$ with an out-of-plane $(z)$ direction (Fig. 1b). An electric field $E_{z}(x, y)$ perpendicular to the crystal plane can then be used to shift the exciton energy by $\delta E=-p_{z} E_{z}$, while its lateral modulation drives the exciton motion towards regions of lower energy. Exciton
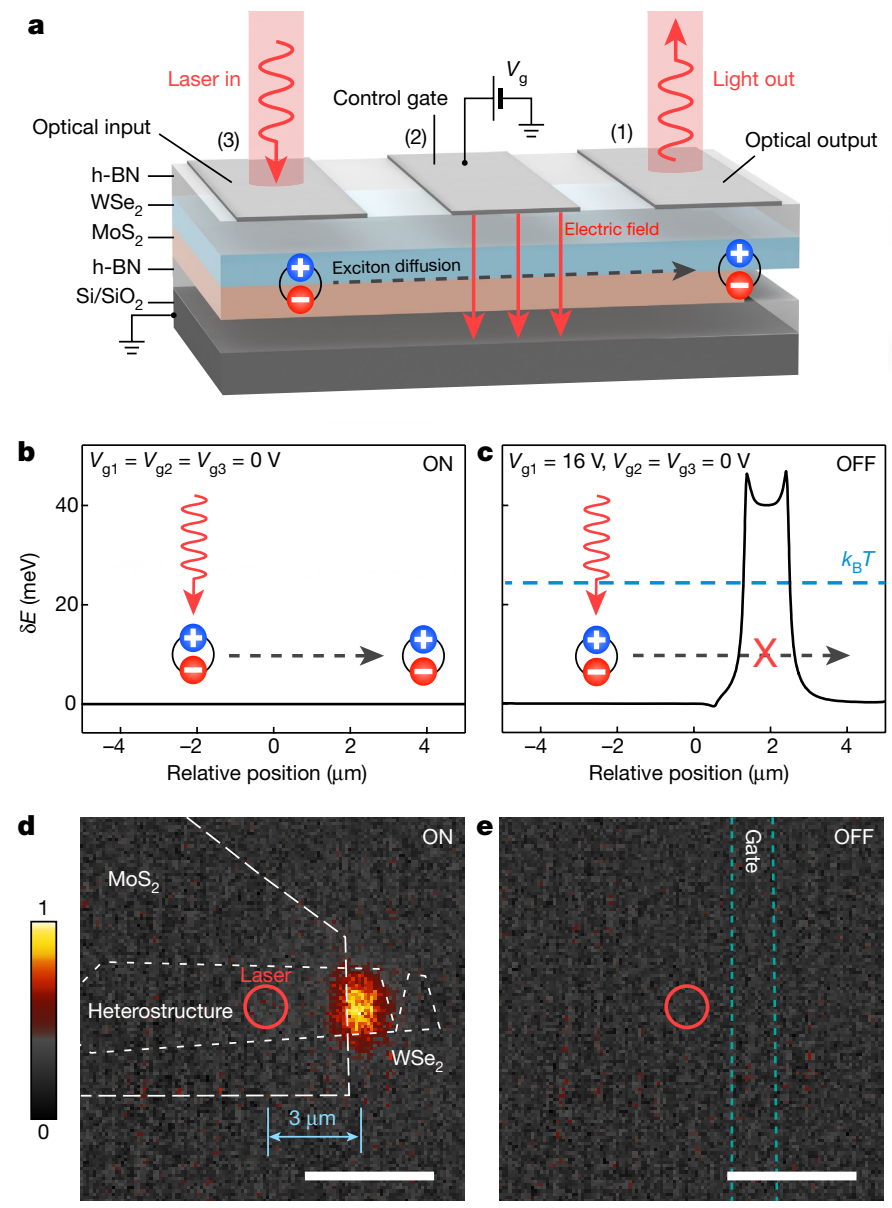

$\mathbf{f}$

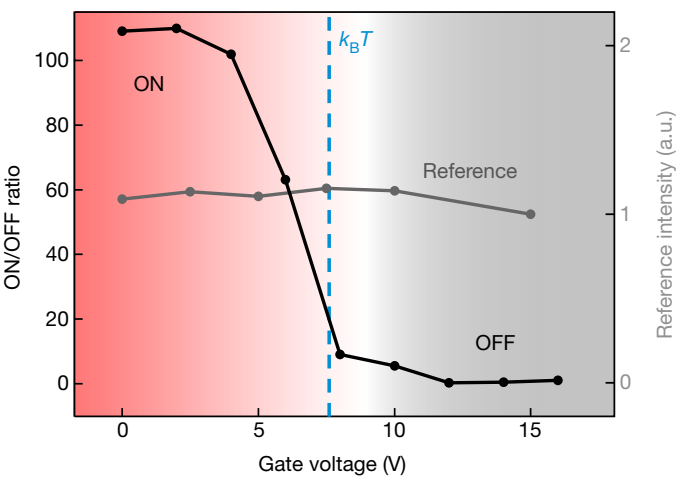

Fig. 2 | Excitonic transistor operation at room temperature. a, The application of gate voltages $\left(V_{\mathrm{g} 1}, V_{\mathrm{g} 2}, V_{\mathrm{g} 3}\right)$ to transparent graphene electrodes (gates 1-3) can engineer a potential landscape for the diffusion of excitons, controlling their flux through the device. $\mathbf{b}, \mathbf{c}$, Calculated energy variation $\delta E$ for the excitons in the $O N$ (free diffusion; b) and OFF (potential barrier; c) states. Red arrows represent laser excitation; the bound charges and black dashed arrows denote the excitons and their diffusion, respectively. d, e, Corresponding images of exciton emission. Dashed lines indicate the positions of the different layers that form the heterostructure and the top graphene gate (gate 1). The laser spot is represented by the red circle. Colour scale indicates the normalized photoluminescence intensity. Scale bars, $5 \mu \mathrm{m}$. f, Gate dependence of the $\mathrm{ON} / \mathrm{OFF}$ ratio for optical excitation $3 \mu \mathrm{m}$ away from the emission centre (left axis). The right axis shows the reference data, which were acquired with the incident laser beam located directly on the emission centre (input-output distance, $\mathrm{d}_{\mathrm{i}-\mathrm{o}}=0 \mu \mathrm{m}$ ). The measured emission intensity is normalized by the OFF-state value at $V_{\mathrm{g} 1}=15 \mathrm{~V}$. The background shading indicates the ON (red) and OFF (grey) states. The blue dashed line represents the gate voltage at which the barrier height is equal to the thermal energy. 

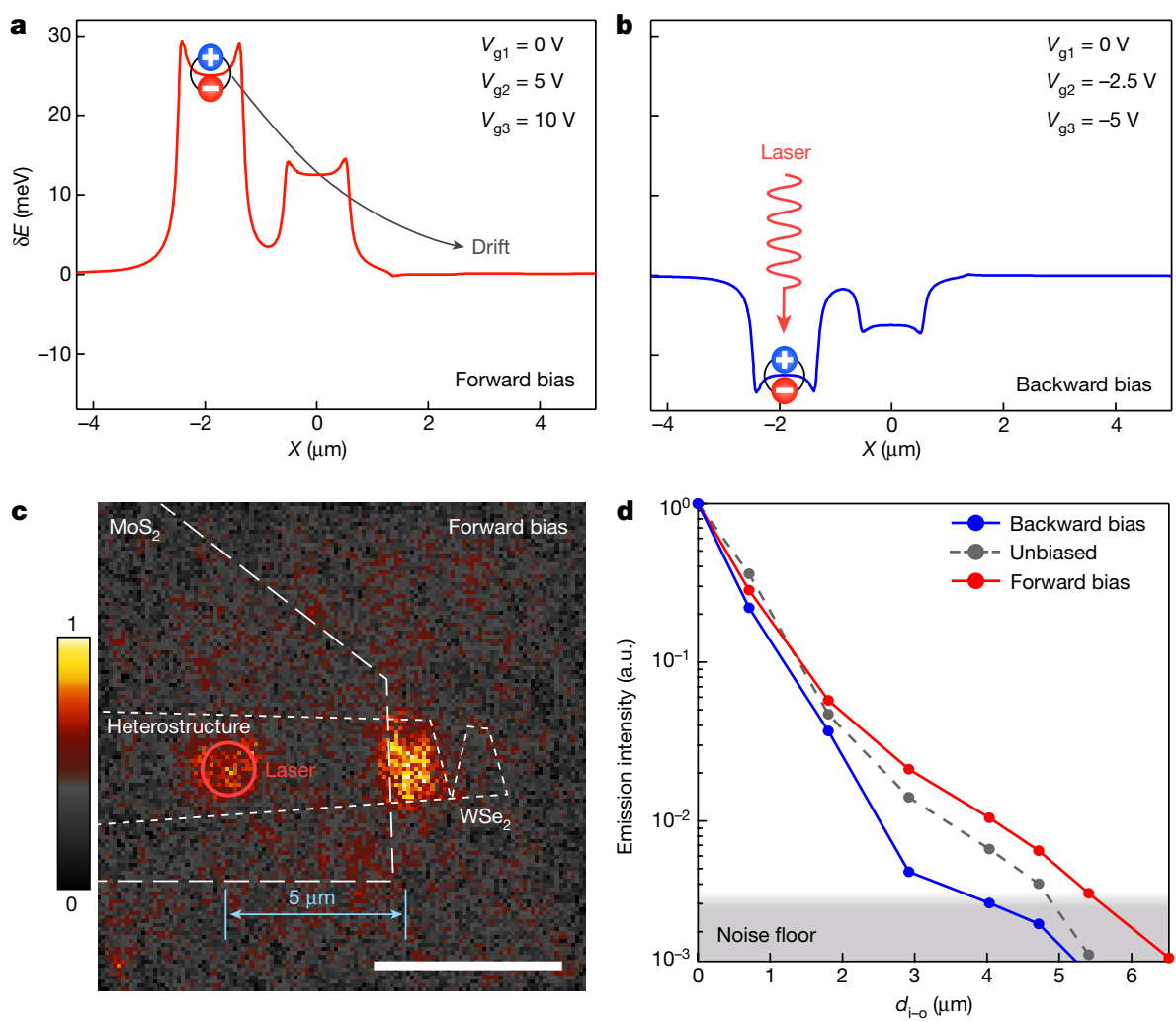

Fig. 3 Biasing of the excitonic device. a, b, Calculated energy profile $\delta E$ of the indirect exciton as a function of lateral coordinate $X$ for the forward (a) and backward (b) bias cases. The black solid line indicates the direction of exciton drift. c, Image showing exciton emission from the device when injecting at a distance $d_{\mathrm{i}-\mathrm{o}}=5 \mu \mathrm{m}$ from the emission area. Colour scale, dashed lines and red circles as in Fig. 2 d, e. Scale bar, $5 \mu \mathrm{m}$. d, Normalized

dynamics in the longitudinal direction can be modelled by a diffusion equation with an external potential (see Methods):

$$
D \frac{\partial^{2} n}{\partial x^{2}}+\frac{D}{k_{\mathrm{B}} T} \frac{\partial}{\partial x}\left(n \frac{\partial \varphi}{\partial x}\right)+G-\frac{n}{\tau}=\frac{\partial n}{\partial t}
$$

where $n, D$ and $\tau$ are the interlayer-exciton concentration, diffusion coefficient and lifetime, respectively, $\varphi$ is the exciton potential (including the electrostatic contribution $\varphi_{\mathrm{el}}=-p_{z} E_{z}$ ) and $G$ is the optical generation rate. This simple model qualitatively shows how the application of an electric field $E_{z}$ can affect interlayer exciton diffusion, as we discuss later.

We first demonstrate an electrically controlled excitonic switch, represented schematically in Fig. 2a. Laser light focused inside the heterostructure area (input) generates interlayer excitons, which diffuse along the channel of the heterostructure. However, the low brightness of interlayer emission makes monitoring the operation of the device challenging. For this reason, we use the exposed $\mathrm{WSe}_{2}$ that extends out of the heterostructure as a bright emitter. Here, interlayer excitons diffuse towards the edge of the heterostructure. During this diffusion process, interlayer excitons are expected to dissociate into single carriers, which are allowed to diffuse inside monolayer $\mathrm{MoS}_{2}$ and $\mathrm{WSe}_{2}$, where they experience recombination with native charges, resulting in bright emission. The emitted radiation is recorded simultaneously using a charge-coupled device (CCD) camera and a spectrometer (see Methods), to obtain spatial and spectral emission profiles. This allows us to further confirm the presence and diffusion of interlayer excitons inside the heterobilayer (Extended Data Fig. 2). In the absence of applied fields (Fig. 2b), excitons diffuse away from the pumping area (red circle in Fig. 2d), owing to temperature and concentration gradients $^{25-27}$, and reach the recombination site, approximately $3 \mu \mathrm{m}$ output intensity as a function of the distance $d_{\mathrm{i}-\mathrm{o}}$ between optical injection and the emission point, for the forward (red) and backward (blue) bias configurations, compared to the unbiased case (grey). The grey shading indicates the noise floor. Exciton diffusion over a distance of $5.5 \mu \mathrm{m}$ is achieved.

away. Comparison of pumping and emission profiles (Extended Data Fig. 4) lets us exclude the possibility of a direct excitation of monolayer $\mathrm{WSe}_{2}$ by the low-intensity tail of the laser spot. This situation (bright output) is shown in the emission image in Fig. $2 \mathrm{~d}$ and corresponds to the ON state of the excitonic transistor. On the contrary, by introducing a potential barrier higher than $k_{\mathrm{B}} T$ on the path of the diffusing excitons (Fig. 2c), we impede their motion, resulting in the suppression of light emission (Fig. 2e). In this way, we can achieve efficient electrical modulation of the output emission, as shown in Fig. 2f, in which the emission intensity (normalized by the value in the OFF state, corresponding to $V_{\mathrm{g} 1}=+16 \mathrm{~V}$ ) is plotted as a function of applied voltage. For reference, we also plot the intensity modulation observed when the laser beam is located on the emission centre (input-output distance $d_{\mathrm{i}-\mathrm{o}}=0 \mu \mathrm{m}$ ). The switching threshold is around $8 \mathrm{~V}$, which corresponds well with the calculated exciton energy modulation of $\delta E \approx k_{\mathrm{B}} T \approx 25 \mathrm{meV}$ (blue dashed line in Fig. 2f). This result is consistent with our model: because the height of the energy barrier starts to become comparable to thermal excitation, it is now possible to block the diffusion of exciton flux. We extract an intensity ON/OFF ratio larger than 100, limited by the noise level of the set-up in the OFF state (see also Extended Data Figs. 4, 5). Such a high ratio results from the realization of an excitonic transistor with complete suppression of emission in the OFF state. This effect is also clearly visible in the spectrum of the emitted light, in which the $\mathrm{WSe}_{2}$ peak is selectively suppressed when the device is in the OFF state (Extended Data Fig. 6). We also note that strong emission from $\mathrm{MoS}_{2}$ is detected in both states, because excitons can diffuse freely in other directions.

An alternative mechanism that could in principle explain the recombination far away from the excitation spot is based on the diffusion of single carriers rather than interlayer excitons. It has been shown that such carriers (holes in particular) can have long lifetimes ${ }^{6,28,29}$. 

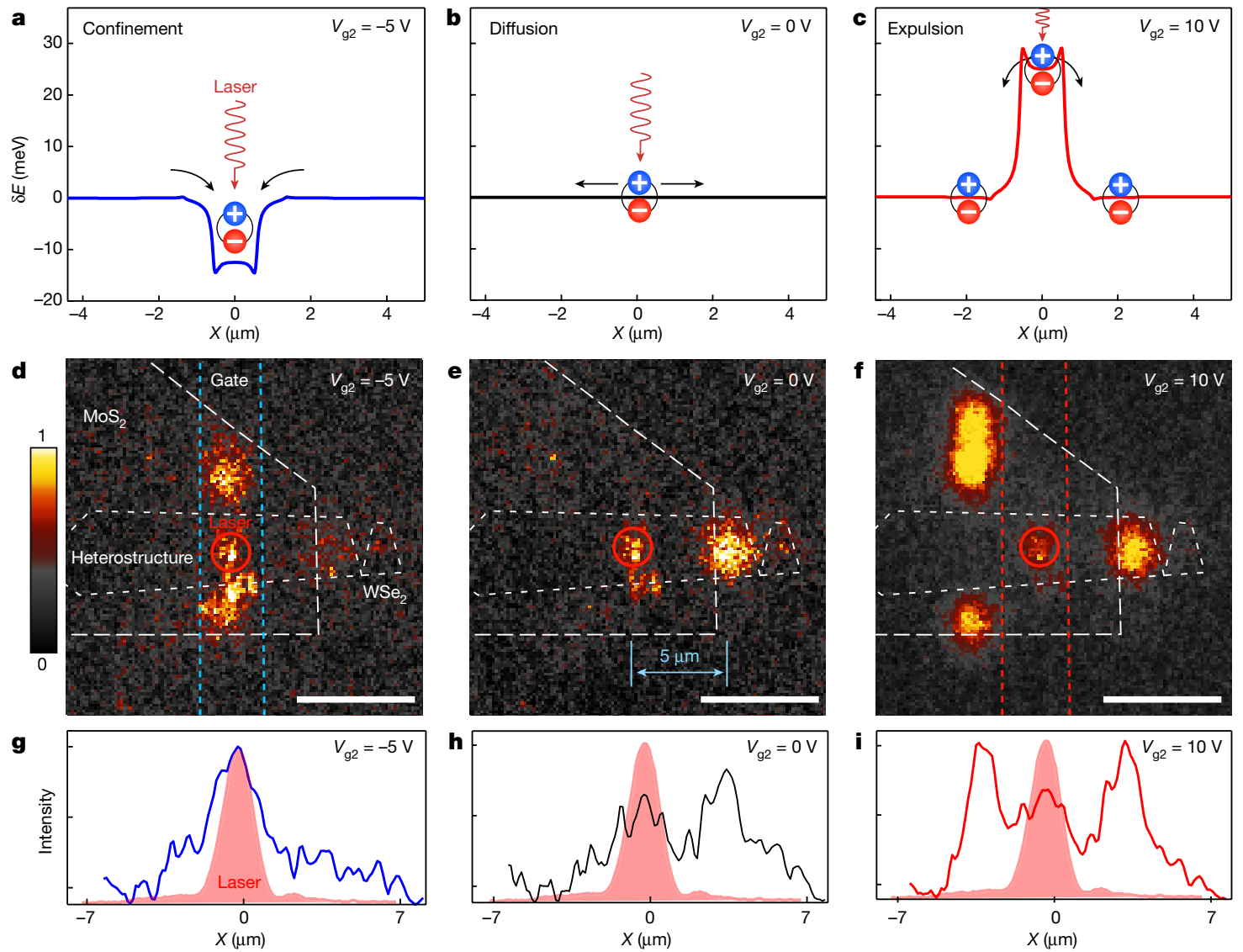

Fig. 4 | Electrically reconfigurable energy landscape. a-c, Calculated energy profile $\delta E$ of the indirect exciton for the cases of a potential well (a), free diffusion (b) and a potential barrier (c). $\mathbf{d}-\mathbf{f}$, Imaging of exciton emission for the configurations shown in $\mathbf{a}-\mathbf{c}$. Incident laser light (red circle) is focused on top of gate 2 . Dashed lines indicate positions of different layers that form the heterostructure and the graphene top gate 2; colour scale as in Fig. 2d, e. Scale bars, $5 \mu \mathrm{m}$. g-i, Cross-section of the intensity profile along the device channel, integrated over its width, for the three configurations. The red-shaded underlay represents the profile of the excitation laser.
However, experimental observations indicate that this is not the dominant mechanism in our heterostructure. First, we observe the production of interlayer excitons directly in the excitation area, even if the intensity is low. Second, for a flux of single carriers, the voltage modulation necessary to counteract thermal excitation and block the single-particle flux would be about $50 \mathrm{mV}$, more than two orders of magnitude lower that the gate voltage of approximately $8 \mathrm{~V}$ required in our experimental result shown in Fig. 2. Finally, this mechanism would also result in different emission profiles for different regimes of device operation (see Extended Data Fig. 7).

To exclude the possibility that the observed effect arises from an unwanted modulation of the charge carrier density in $\mathrm{WSe}_{2}$, we perform a calibration experiment in which the excitation light is focused on the output area $\left(d_{\mathrm{i}-\mathrm{o}}=0\right)$ and the device is biased as before. This reference experiment is discussed in detail in Methods and the result is presented in Fig. $2 \mathrm{f}$ (grey curve); it shows that only a comparably small modulation of $\mathrm{WSe}_{2}$ emission intensity is observed. This confirms that the energy barrier is the origin of the switching behaviour. We study the dependence of the ON/OFF ratio on $d_{\mathrm{i}-\mathrm{o}}$ further (Extended Data Fig. 8) by keeping the voltage profile constant and optically injecting excitons at different distances from the output point. Consistent with our model, we observe efficient modulation when the laser is focused beyond the energy barrier, with emission intensity decreasing with increasing $d_{\mathrm{i}-\mathrm{o}}$ owing to long-distance diffusion. The diffusion length can be doubled at lower temperature $(4.7 \mathrm{~K})$, resulting in operation over a longer distance (Extended Data Fig. 9).

Having demonstrated that we can block or allow spontaneous exciton diffusion, we go further by creating a drift field in the desired direction, in analogy with the source-drain bias of a conventional FET. We show this type of operation in Fig. 3, with all three electrodes used to create a potential ladder going upwards or downwards with respect to the excitation point (Fig. 3a, b). When excitons encounter a gradually decreasing energy profile (forward bias), their diffusion is enhanced by a drift term, allowing us to operate the device with a larger distance between optical input and output. As shown in Fig. 3c, this regime of electrically assisted diffusion can result in exciton transport over a distance of $5 \mu \mathrm{m}$. To obtain a more quantitative estimate of the induced modulation, we measure the dependence of the emission intensity on the distance from the laser spot as it is displaced away from the output area at fixed gate voltages. The results (Fig. 3d) show that the length over which excitons diffuse can be effectively modulated from $5.5 \mu \mathrm{m}$ to $3 \mu \mathrm{m}$, compared to about $4.5 \mu \mathrm{m}$ in the unbiased case. The modulation of the effective diffusion length with the potential $\varphi_{\mathrm{el}}$ qualitatively follows the model introduced in equation (1).

We further use the multi-gate configuration to demonstrate more complex and electrically reconfigurable types of potential landscape and related device operation. In Fig. $4 \mathrm{a}-\mathrm{c}$ we present the energy profiles calculated for free diffusion (Fig. 4b) compared with a potential well (Fig. 4a) and a repulsive barrier (Fig. 4c) produced by the central gate (gate 2 ), while the side gates ( 1 and 3 ) are kept grounded. In this case, the position of the optical pump is centred on the middle electrode, which corresponds to the centre of the well or barrier. In Fig. 4d, g we show the CCD camera image and related emitted intensity profile along the device channel for the case of the potential well. We observe photoluminescence emission only from the narrow area below the central contact, which is indicative of electrical confinement of the excitonic cloud. Conversely, when applying a positive voltage to create a 'potential hill' (Fig. 4f, i), we see an expulsion of excitons from the pumping area 
with the appearance of bright emission spots outside the middle section of the device, owing to excitons drifting along the energy profile and recombining on the edges of the heterostructure. This is evident from a comparison with the free-diffusion case in Fig. 4e, h. Interestingly, we also observe higher-energy emission from the neighbouring $\mathrm{MoS}_{2}$ monolayer parts inside the well in the case of exciton confinement. A similar effect is also observed during exciton expulsion, with bright spots appearing at the edges of the heterostructure around the repulsive potential. Further inspection of the emission spectra from Fig. $4 \mathrm{~d}, \mathrm{f}$ confirms this, with the intensity of monolayer peaks decreasing (increasing) when confining (anti-confining) the excitons (Extended Data Fig. 6). As also discussed in Methods, the observed $\mathrm{MoS}_{2}$ emission is affected by the local inhomogeneity of the substrate and by the optical filters used. As discussed earlier, the diffusion of single particles and their recombination with native charges that are available in the monolayers could have a role in light emission that extends from the edges of the heterobilayer into the monolayers.

\section{Online content}

Any Methods, including any statements of data availability and Nature Research reporting summaries, along with any additional references and Source Data files, are available in the online version of the paper at https://doi.org/10.1038/s41586018-0357-y

Received: 29 November 2017; Accepted: 4 June 2018;

Published online: 25 July 2018

1. High, A. A., Hammack, A. T., Butov, L. V., Hanson, M. \& Gossard, A. C. Exciton optoelectronic transistor. Opt. Lett. 32, 2466-2468 (2007).

2. High, A. A., Novitskaya, E. E., Butov, L. V., Hanson, M. \& Gossard, A. C. Control of exciton fluxes in an excitonic integrated circuit. Science 321, 229-231 (2008).

3. Grosso, G. et al. Excitonic switches operating at around 100 K. Nat. Photon. 3, 577-580 (2009).

4. Cheiwchanchamnangij, T. \& Lambrecht, W. R. L. Quasiparticle band structure calculation of monolayer, bilayer, and bulk MoS2. Phys. Rev. B 85, 205302 (2012).

5. He, K. et al. Tightly bound excitons in monolayer WSe 2 . Phys. Rev. Lett. 113 026803 (2014).

6. Rivera, P. et al. Observation of long-lived interlayer excitons in monolayer $\mathrm{MoSe}_{2}-\mathrm{WSe}_{2}$ heterostructures. Nat. Commun. 6, 6242 (2015).

7. Palummo, M., Bernardi, M. \& Grossman, J. C. Exciton radiative lifetimes in twodimensional transition metal dichalcogenides. Nano Lett. 15, 2794-2800 (2015).

8. Miller, B. et al. Long-lived direct and indirect interlayer excitons in van der Waals heterostructures. Nano Lett. 17, 5229-5237 (2017).

9. Žutić, I., Fabian, J. \& Das Sarma, S. Spintronics: fundamentals and applications. Rev. Mod. Phys. 76, 323-410 (2004).

10. Miller, D. A. B. Rationale and challenges for optical interconnects to electronic chips. Proc. IEEE 88, 728-749 (2000).

11. Butov, L. V. Excitonic devices. Superlattices Microstruct. 108, 2-26 (2017).

12. Liu, A. et al. A high-speed silicon optical modulator based on a metal-oxidesemiconductor capacitor. Nature 427, 615-618 (2004)

13. Melikyan, A. et al. High-speed plasmonic phase modulators. Nat. Photon. 8 , 229-233 (2014).

14. Xu, Q., Schmidt, B., Pradhan, S. \& Lipson, M. Micrometre-scale silicon electro-optic modulator. Nature 435, 325-327 (2005).
15. Schmidt, B., Xu, Q., Shakya, J., Manipatruni, S. \& Lipson, M. Compact electro-optic modulator on silicon-on-insulator substrates using cavities with ultra-small modal volumes. Opt. Express 15, 3140-3148 (2007).

16. Fedichkin, F. et al. Room-temperature transport of indirect excitons in AIGaN/ GaN quantum wells. Phys. Rev. Appl. 6, 014011 (2016).

17. Kuznetsova, Y. Y. et al. Transport of indirect excitons in $\mathrm{ZnO}$ quantum wells. Opt. Lett. 40, 3667-3670 (2015).

18. Fogler, M. M., Butov, L. V. \& Novoselov, K. S. High-temperature superfluidity with indirect excitons in van der Waals heterostructures. Nat. Commun. 5, 4555 (2014).

19. Robert, C. et al. Exciton radiative lifetime in transition metal dichalcogenide monolayers. Phys. Rev. B 93, 205423 (2016).

20. Kang, J., Tongay, S., Zhou, J., Li, J. \& Wu, J. Band offsets and heterostructures of two-dimensional semiconductors. Appl. Phys. Lett. 102, 012111 (2013).

21. Chiu, M.-H. et al. Determination of band alignment in the single-layer $\mathrm{MoS}_{2} /$ WSe 2 heterojunction. Nat. Commun. 6, 7666 (2015).

22. Fang, $\mathrm{H}$. et al. Strong interlayer coupling in van der Waals heterostructures built from single-layer chalcogenides. Proc. Natl Acad. Sci. USA 111, 6198-6202 (2014).

23. Kunstmann, J. et al. Momentum-space indirect interlayer excitons in transition-metal dichalcogenide van der Waals heterostructures. Nat. Phys. https://doi.org/10.1038/s41567-018-0123-y (2018).

24. Kim, J. et al. Observation of ultralong valley lifetime in $\mathrm{WSe}_{2} / \mathrm{MoS} 2$ heterostructures. Sci. Adv. 3, e1700518 (2017).

25. Cadiz, F. et al. Exciton diffusion in WSe $\mathrm{S}_{2}$ monolayers embedded in a van der Waals heterostructure. Appl. Phys. Lett. 112, 152106 (2018).

26. Kulig, M. et al. Exciton diffusion and halo effects in monolayer semiconductors. Phys. Rev. Lett. 120, 207401 (2018)

27. Onga, M., Zhang, Y., Ideue, T. \& Iwasa, Y. Exciton Hall effect in monolayer MoS2. Nat. Mater. 16, 1193-1197 (2017).

28. Nagler, P. et al. Interlayer exciton dynamics in a dichalcogenide monolayer heterostructure. 2D Mater. 4, 025112 (2017).

29. Wang, R. et al. Ultrafast and spatially resolved studies of charge carriers in atomically thin molybdenum disulfide. Phys. Rev. B 86, 045406 (2012).

Acknowledgements We are grateful to K. Marinov and D. Ovchinnikov for discussion. We acknowledge the help of Z. Benes (CMI) with electron-beam lithography. D.U., A.C., A.A. and A.K. acknowledge support by the Swiss National Science Foundation (grant 153298), H2020 European Research Council (ERC, grant 682332) and Marie Curie-Sklodowska-Curie Actions (COFUND grant 665667). A.K. acknowledges funding from the European Union's Horizon H2020 Future and Emerging Technologies under grant agreement number 696656 (Graphene Flagship). K.W. and T.T. acknowledge support from the Elemental Strategy Initiative conducted by the MEXT, Japan and JSPS KAKENHI grant numbers JP15K21722 and JP25106006.

Reviewer information Nature thanks W. Gao, A. Tartakovskii and the other anonymous reviewer(s) for their contribution to the peer review of this work

Author contributions A.C. and A.A. fabricated the devices, D.U. and A.C. performed the optical measurements and analysed the data. K.W. and T.T. grew the h-BN crystals. A.K. initiated and supervised the project. D.U., A.C., A.A. and A.K. wrote the manuscript with input from all authors.

Competing interests The authors declare no competing interests.

Additional information

Extended data is available for this paper at https://doi.org/10.1038/s41586018-0357-y.

Reprints and permissions information is available at http://www.nature.com/ reprints.

Correspondence and requests for materials should be addressed to A.K.

Publisher's note: Springer Nature remains neutral with regard to jurisdictional claims in published maps and institutional affiliations. 


\section{METHODS}

Device fabrication. The heterostructure was fabricated using polymer-assisted transfer (see Extended Data Fig. 10) of flakes of hexagonal boron nitride (h-BN), $\mathrm{WSe}_{2}$ (HQ Graphene) and $\mathrm{MoS}_{2}$ (SPI). Flakes were first exfoliated on a polymer double layer, as described previously ${ }^{30}$. Once monolayers were optically identified, the bottom layer was dissolved with a solvent and free-floating films with flakes were obtained. These were transferred using a custom-built set-up with micromanipulators to carefully align flakes on top of each other. During the transfer process, the sharp edges of the flakes were aligned to obtain a twist angle between the two crystal axes close to $0^{\circ}\left(\right.$ or $\left.60^{\circ}\right)$. However, in the case of $\mathrm{MoS}_{2}-\mathrm{WSe}_{2}$ heterobilayers, the alignment has been shown to be not critical for the observation of interlayer excitons ${ }^{23,31}$. This is due to the indirect (in reciprocal space) nature of the transition and to the considerable lattice mismatch between the two layers (about 4\%). Polymer residue was removed with a hot acetone bath. Once completed, the stack was thermally annealed in high vacuum at $10^{-6} \mathrm{mbar}$ for $6 \mathrm{~h}$. Few-layer graphene flakes were obtained by exfoliation from graphite (NGS) on $\mathrm{Si} / \mathrm{SiO}_{2}$ substrates and patterned in the desired shape by electron-beam lithography and oxygen plasma etching. After thermal annealing, the patterned flakes were transferred on top of the van der Waals stack using a polymer-assisted transfer and the entire structure was annealed again in high vacuum. Finally, electrical contacts were fabricated by electron-beam lithography and metallization $(60 \mathrm{~nm} / 2 \mathrm{~nm} \mathrm{Au} / \mathrm{Ti})$.

Optical measurements. All measurements presented here were performed in vacuum at room temperature unless specified otherwise. Excitons were optically pumped by a continuous-wave 647 -nm laser diode focused to the diffraction limit with a beam size of about $1 \mu \mathrm{m}$. The incident power was $250 \mu \mathrm{W}$. The spectral and spatial characteristics of the device emission were analysed simultaneously. The emitted light was acquired using a spectrometer (Andor) and the laser line was removed with a long-pass 650 -nm edge filter. For spatial imaging, we used a long-pass 700-nm edge filter so that the laser light and most of the $\mathrm{MoS}_{2}$ emission were blocked. Filtered light was acquired by a CCD camera (Andor Ixon). The room-temperature photoluminescence spectrum of $\mathrm{MoS}_{2}$ shown in Extended Data Fig. $1 \mathrm{~b}$ was obtained under $150-\mu \mathrm{W}$ excitation at $647 \mathrm{~nm}$, whereas monolayer $\mathrm{WSe}_{2}$ and the heterostructure fabricated on $\mathrm{SiO}_{2}$ substrate were characterized under 488-nm excitation.

Owing to the small separation between the interlayer and the intralayer $\mathrm{WSe}_{2}$ exciton peaks, it is not possible to completely distinguish them in the images acquired on the CCD. The tail of the $\mathrm{WSe}_{2}$ monolayer peak normally overlaps with the spectral line of the interlayer exciton considerably, meaning that weak luminescence around $785 \mathrm{~nm}$ can be observed even on monolayer WSe $\mathrm{W}_{2}$ (Extended Data Fig. 3e), which is not due to interlayer excitons.

Because of the use of the 700-nm filter, the emission from monolayer $\mathrm{MoS}_{2}$ is in principle not observable on the CCD. However, some light can be transmitted when the broadening of the photoluminescence peak results in a low-energy tail (see Extended Data Fig. 11) extending beyond $700 \mathrm{~nm}$. Local inhomogeneity in the substrate can affect this broadening, which could explain why the observed $\mathrm{MoS}_{2}$ luminescence in Fig. $4 \mathrm{f}$ comes mostly from the left part of the device.

Low-temperature measurements (Extended Data Fig. 9) were performed in a liquid-helium, continuous-flow cryostat (Oxford Instruments).

Reference experiment. We performed a reference experiment to exclude spurious effects that could compromise the interpretation of the data. First, we observed how the photoluminescence emission from monolayer $\mathrm{WSe}_{2}$ changes when gating the device using the back gate. For this purpose, we excited the exposed $\mathrm{WSe}_{2}$ with the laser beam directly and recorded the photoluminescence spectra. When applying voltage to the back gate, a modulation in the emission intensity is clearly observable (Extended Data Fig. 12a). We repeated the same measurement, but instead of applying a voltage between the flake and the back gate, we biased the top and back gates, thus generating a vertical electric field inside the device. In this case, we cannot observe any substantial change in the emission intensity (Extended Data Fig. 12b). This allows us to rule out the possibility that the switching action that we observe could be due to a suppression of photoluminescence from a changing doping level in the material.

Image processing. To aid the interpretation of images from the CCD camera, we performed several image-processing steps using Image ${ }^{32}$. We first subtracted from the original image a background image obtained without laser illumination, to account for ambient light noise. In some cases, a simple background was not sufficient to compensate for the presence of spurious signals from unwanted reflections or changing ambient background. In these cases, a background image was generated by applying the rolling-ball algorithm in ImageJ. Contrast was adjusted to cover the range of values in the image. We provide an example of the procedure in Extended Data Fig. 13.

Modelling exciton diffusion. The dynamics of the exciton in the channel of our device can be modelled by one-dimensional diffusion in the presence of an external potential $\varphi(x)$ (temperature, electrostatic potential or dipole-dipole interaction). The gradient of exciton concentration $n(x)$ drives diffusion current $j_{\text {diff }}$ while the potential gradient causes drift $j_{\text {drift: }}$

$$
j_{\text {diff }}=-D \frac{\partial n}{\partial x}, j_{\text {drift }}=-\mu n \frac{\partial \varphi}{\partial x}
$$

where $\mu$ is the exciton mobility, which is related to the diffusion coefficient $D$ and the thermal energy $k_{\mathrm{B}} T$ by the Einstein relation $D=\mu k_{\mathrm{B}} T$. We also include an exciton generation rate $G$ by means of optical pumping and an exciton recombination rate $R$, which is related to the exciton lifetime as $R=-n / \tau$. From the exciton continuity equation we then obtain equation (1).

In our system, in which excitons have a built-in vertical dipole moment $p$, the electrostatic potential induced by the vertical electric field is $\varphi_{\mathrm{el}}=-E_{z} p_{z}$. Because we use continuous-wave excitation, we assume a steady-state case $(\partial n / \partial t=0)$. Considering $\varphi_{\mathrm{el}}$ as the main contribution to exciton drift, we obtain

$$
D \frac{\partial^{2} n}{\partial x^{2}}-\frac{D p_{z}}{k_{\mathrm{B}} T} \frac{\partial}{\partial x}\left(n \frac{\partial E_{z}}{\partial x}\right)+G-\frac{n}{\tau}=0
$$

We simplify the model further by assuming two fundamentally different regions, shown in Extended Data Fig. 14. First region is under constant homogeneous excitation so that the concentration reaches an equilibrium value with equal recombination and generation rates $(R+G=0)$. The equilibrium concentration is then $n_{0}=G \tau$. Outside of the pumping region, excitons diffuse away, driven by the concentration and potential gradients:

$$
D \frac{\partial^{2} n}{\partial x^{2}}-\frac{D p_{z}}{k_{\mathrm{B}} T} \frac{\partial}{\partial x}\left(n \frac{\partial E_{z}}{\partial x}\right)-\frac{n}{\tau}=0
$$

The case of diffusion in the absence of an external field can be solved analytically, revealing exponential decay of exciton density from the pumping region with a characteristic distance that corresponds to the diffusion length $l_{\text {diff }}=\sqrt{D \tau}$ : $n_{\text {free }}(x)=n_{0} \mathrm{e}^{-x / l_{\text {diff }}}$

An applied non-homogeneous vertical electric field can alter the diffusion length (as demonstrated experimentally), which can be modelled as a change in the effective diffusion length.

Numerical simulation of the exciton-energy profile. We first calculate the electricfield distribution in our system using the COMSOL Multiphysics simulation software. All calculations were performed considering the dimensions of the device as follows: the top graphene gates are $1.1 \mu \mathrm{m}$ wide and spaced $0.8 \mu \mathrm{m}$ apart. The heterostructure is encapsulated between two h-BN crystals $(10 \mathrm{~nm}$ thick on the top and $20 \mathrm{~nm}$ on the bottom), and the substrate is heavily doped Si with $270 \mathrm{~nm}$ of $\mathrm{SiO}_{2}$ on top (see Extended Data Fig. 15a). Extended Data Fig. 15b shows an example of the electrical field in the system in the confinement configuration, with $-10 \mathrm{~V}$ applied to the central gate and the side gates grounded. Interlayer excitons have a built-in out-of-plane dipole moment directed upwards, with an absolute value of $p_{z}=e d=e \times 7.5 \times 10^{-10} \mathrm{~m}$, where $e$ is the elementary charge and $d=7.5 \AA$ is the layer separation in our heterostructure. They thus experience an energy shift of $\delta E=-p_{z} E_{z}$ in the presence of a vertical electric field $E_{z}$. The resulting force applied on the exciton in the longitudinal direction is proportional to the first derivative of the vertical electric field $E_{z}$ with respect to the channel $x$ axis:

$$
F_{x}=-\frac{\partial(\delta E)}{\partial x}=e d \frac{\partial E_{z}}{\partial x}
$$

Example profiles of the confinement-well configuration are shown in Extended Data Fig. 15c.

Data availability. The data that support the findings of this study are available from the corresponding author on reasonable request.

30. Mayorov, A. S. et al. Micrometer-scale ballistic transport in encapsulated graphene at room temperature. Nano Lett. 11, 2396-2399 (2011).

31. Zhu, H. et al. Interfacial charge transfer circumventing momentum mismatch at two-dimensional van der Waals heterojunctions. Nano Lett. 17, 3591-3598 (2017).

32. Schneider, C. A., Rasband, W. S. \& Eliceiri, K. W. NIH Image to ImageJ: 25 years of image analysis. Nat. Methods 9, 671-675 (2012). 
a

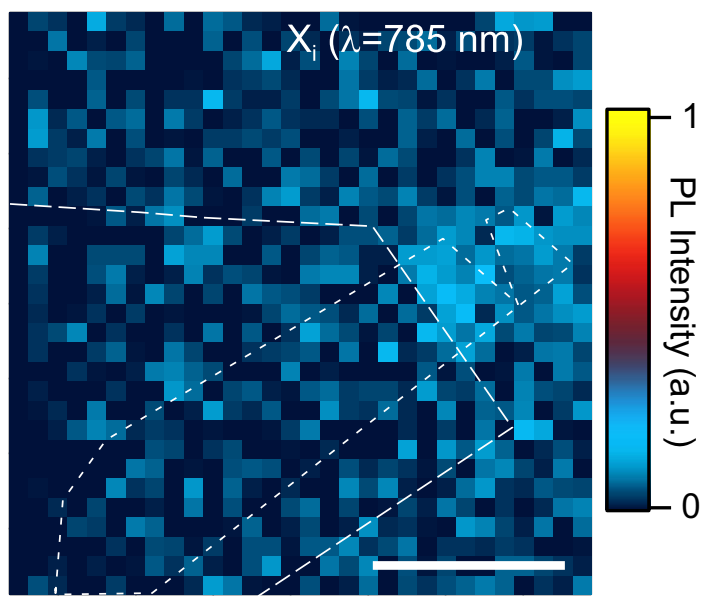

Extended Data Fig. 1 Interlayer excitons in the $\mathrm{WSe}_{2}-\mathrm{MoS}_{2}$ van der Waals heterostructure. a, Spatial map of photoluminescence at $785 \mathrm{~nm}$ corresponding to the heterostructure interlayer photoluminescence emission maximum, as shown in the photoluminescence (PL) spectra in b

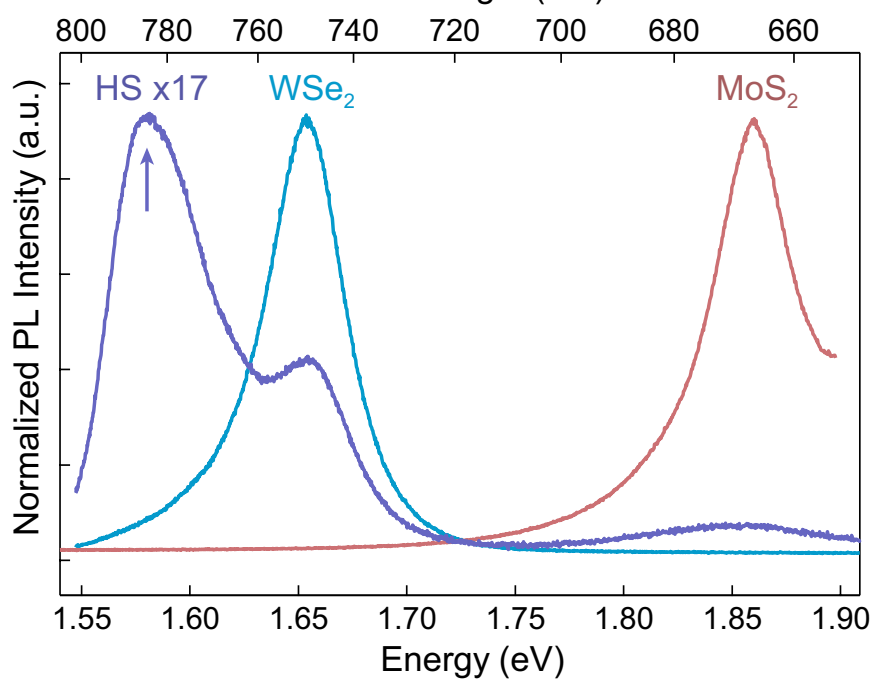

b. An efficient interlayer charge-transfer process in the heterostructure encapsulated in h-BN results in further quenching of photoluminescence emission from the heterostructure. Scale bar, $5 \mu \mathrm{m}$. b. Photoluminescence spectra from the structure fabricated on $\mathrm{SiO}_{2}$. 


\section{RESEARCH LETTER}
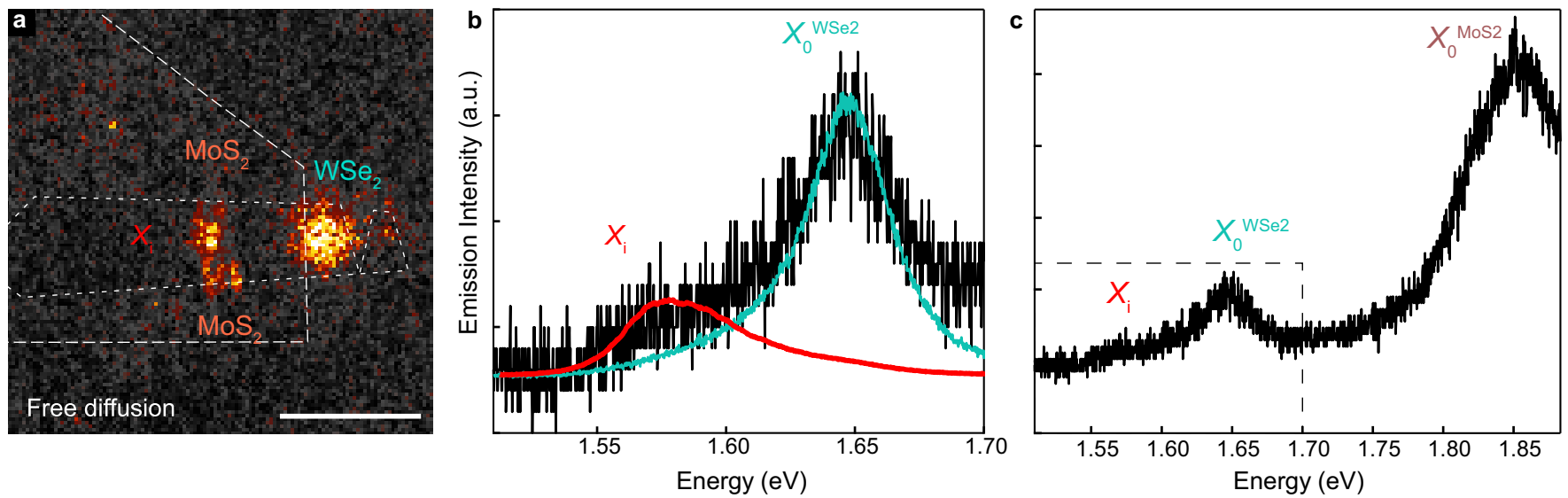

Extended Data Fig. 2 | Spectra of excitonic device emission.

a, Distribution of photoluminescence emission intensity from the device, in the absence of an electric field. White dashed lines represent edges of constituent crystals. Scale bar, $5 \mu \mathrm{m}$. b. Detailed spectrum of the emission pattern, showing the interlayer exciton peak $\left(X_{\mathrm{i}}\right)$ and $\mathrm{WSe}_{2}$ intralayer

emission. We note that the low-energy peak $\left(X_{\mathrm{i}}\right)$ cannot be related to localized excitons in $\mathrm{WSe}_{2}$, because they are observed only at cryogenic temperatures. c, Full spectrum of the emission shown in a, also showing the emission from $\mathrm{MoS}_{2}\left(X_{0}^{\mathrm{MoS}_{2}}\right)$, which is blocked by the filter in the CCD image. The black dashed box indicates the range of energies shown in $\mathbf{b}$. 

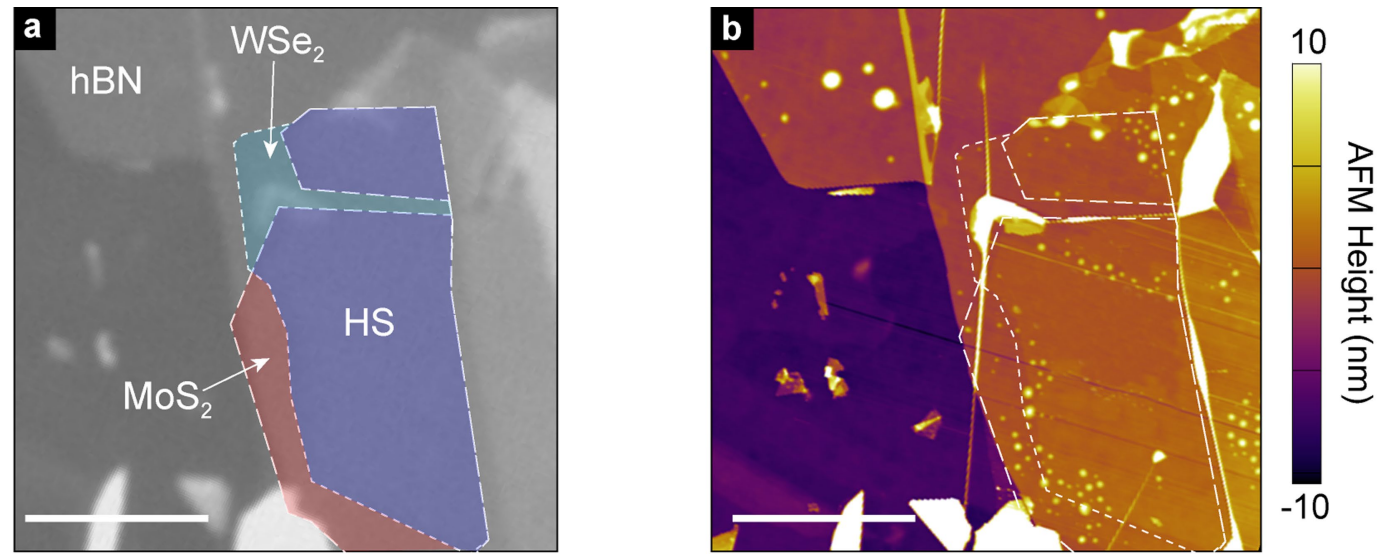

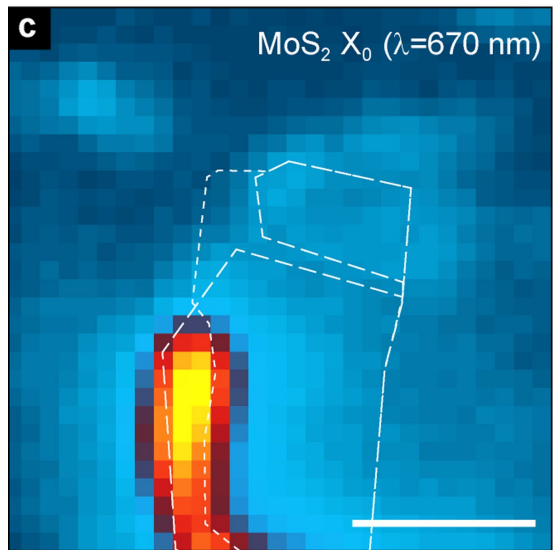

Extended Data Fig. 3 | Characterization of an additional WSe $\mathrm{W}_{2}-\mathrm{MoS}_{2}$ heterostructure. a, False-colour optical image of the fabricated stack. b. Atomic force microscopy (AFM) height-profile image of the heterostructure. $\mathbf{c}-\mathbf{e}$, Spatial maps of photoluminescence intensity at emission wavelengths $(\lambda)$ of $670 \mathrm{~nm}(\mathbf{c}), 750 \mathrm{~nm}(\mathbf{d})$ and $785 \mathrm{~nm}$
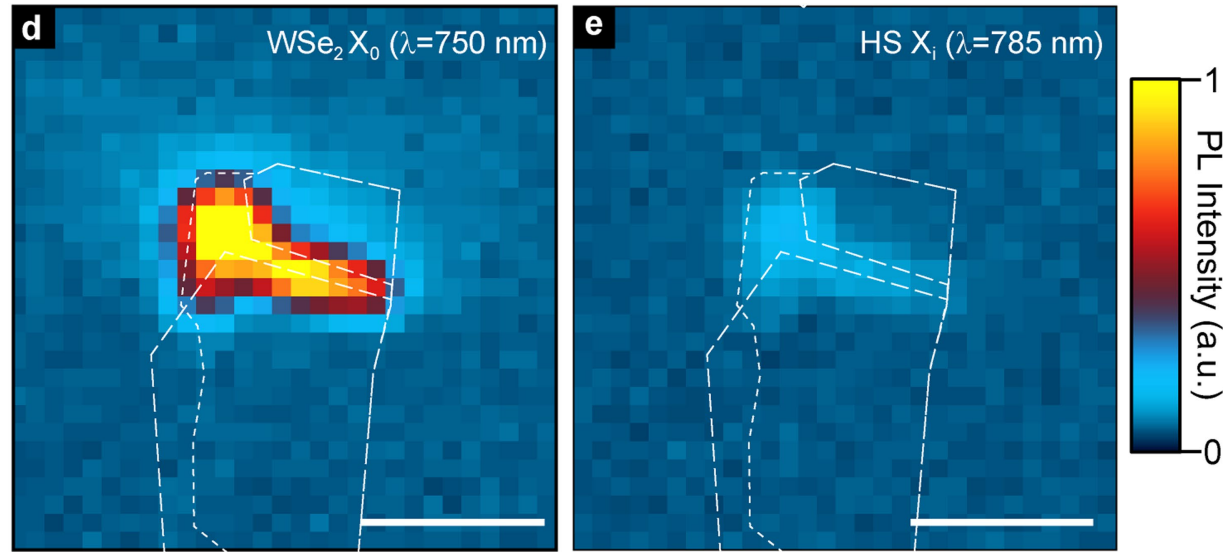

(e), corresponding to $\mathrm{MoS}_{2}$ intralayer $\left(X_{0}\right), \mathrm{WSe}_{2}$ intralayer $\left(X_{0}\right)$ and heterostructure interlayer $\left(X_{\mathrm{i}}\right)$ excitonic resonances. Photoluminescence is quenched in the heterostructure area owing to efficient charge transfer. White dashed lines represent edges of constituent crystals. Scale bars, $5 \mu \mathrm{m}$. 
a

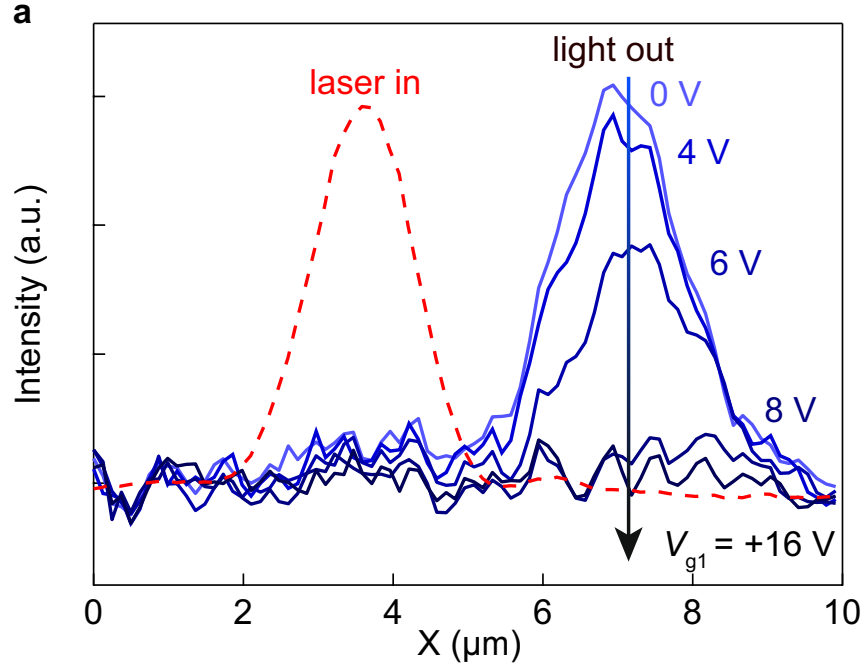

b

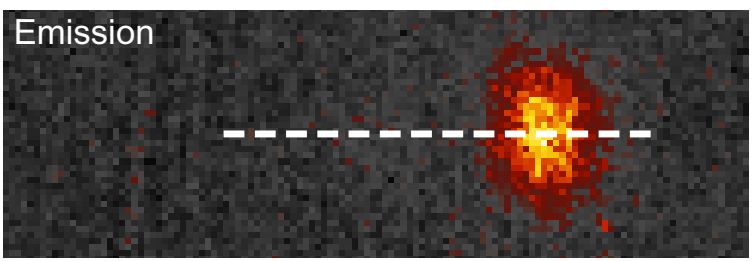

\section{Laser}

Extended Data Fig. 4 | Excitonic transistor input and output. a, Crosssectional profile of the device emission intensity along the white dashed lines in $\mathbf{b}$ and $\mathbf{c}$, obtained for different gate voltages $V_{\mathrm{g} 1}$ from $0 \mathrm{~V}$ (light blue) to $16 \mathrm{~V}$ (black) with intermediate values of $4 \mathrm{~V}, 6 \mathrm{~V}$ and $8 \mathrm{~V}$. The red line represents the intensity profile of the laser spot. b, c, CCD images of the exciton emission in the ON state (b) and of the focused laser spot (c). The lengths of the dashed lines indicate $10 \mu \mathrm{m}$. 
a

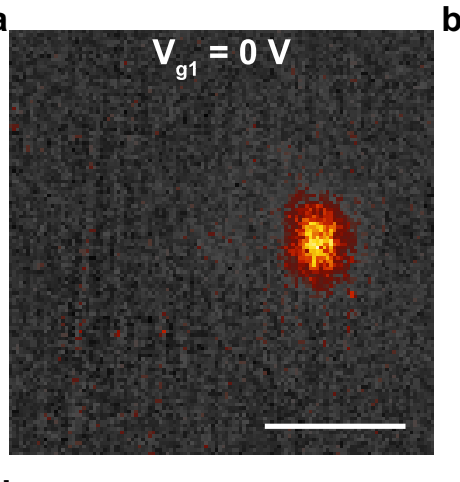

d
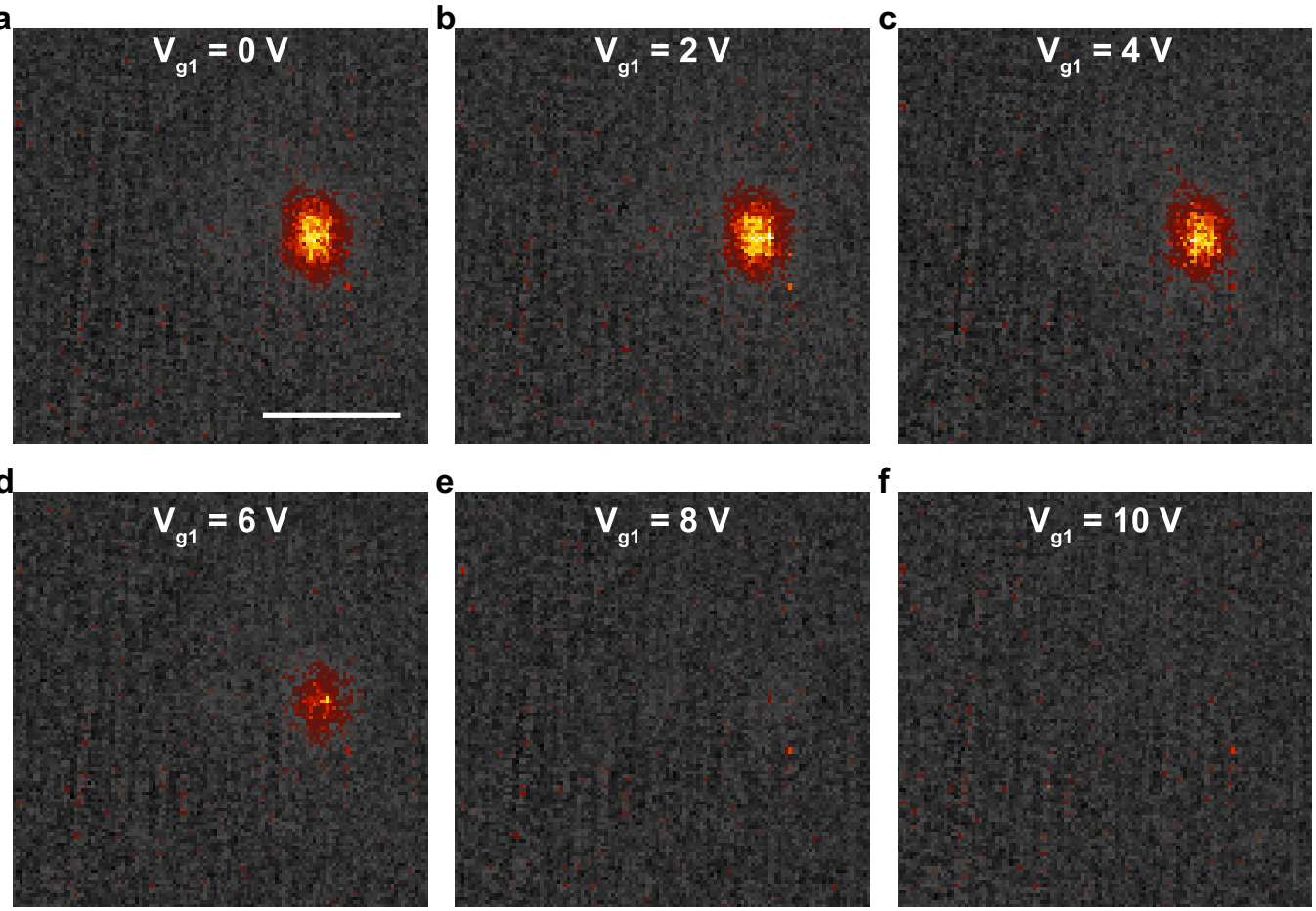

Extended Data Fig. 5 | Switching of the excitonic transistor. a-f, CCD images of exciton emission from the device, obtained for different gate voltages $V_{\mathrm{g} 1}$ from 0 to $10 \mathrm{~V}$ in steps of $2 \mathrm{~V}$.
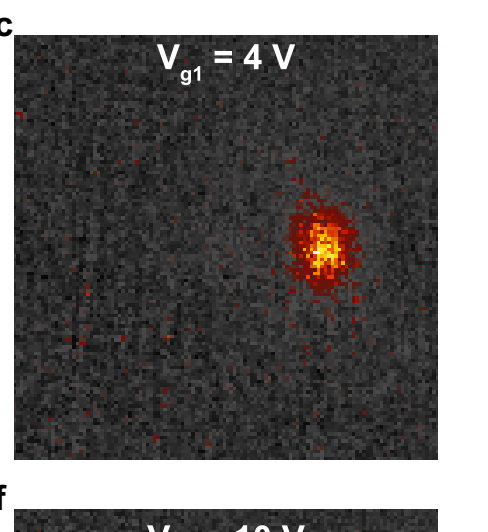
a
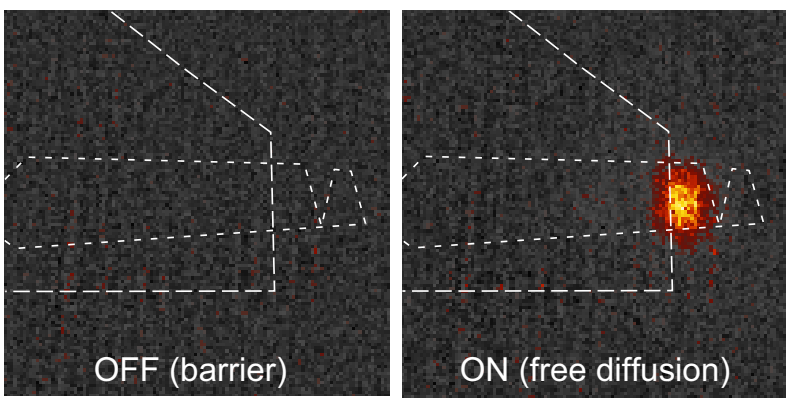

b

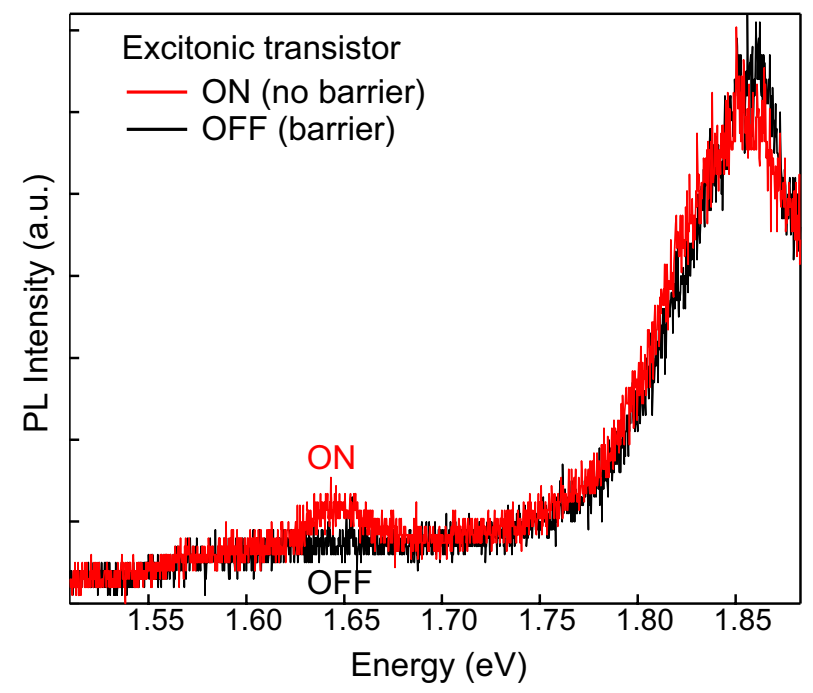

Extended Data Fig. 6 | Spectrum of light emitted from the device in different states. a, b, Intensity distribution of light emission from the excitonic transistor in the OFF and ON states (left and right, respectively; a) and the corresponding spectra collected from the entire device (black
C
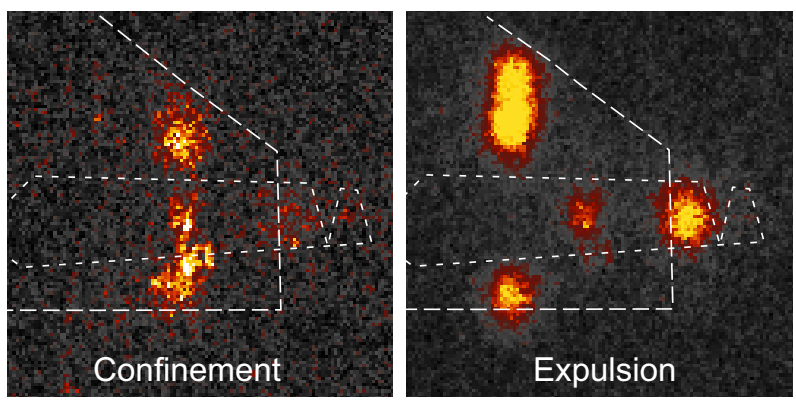

d

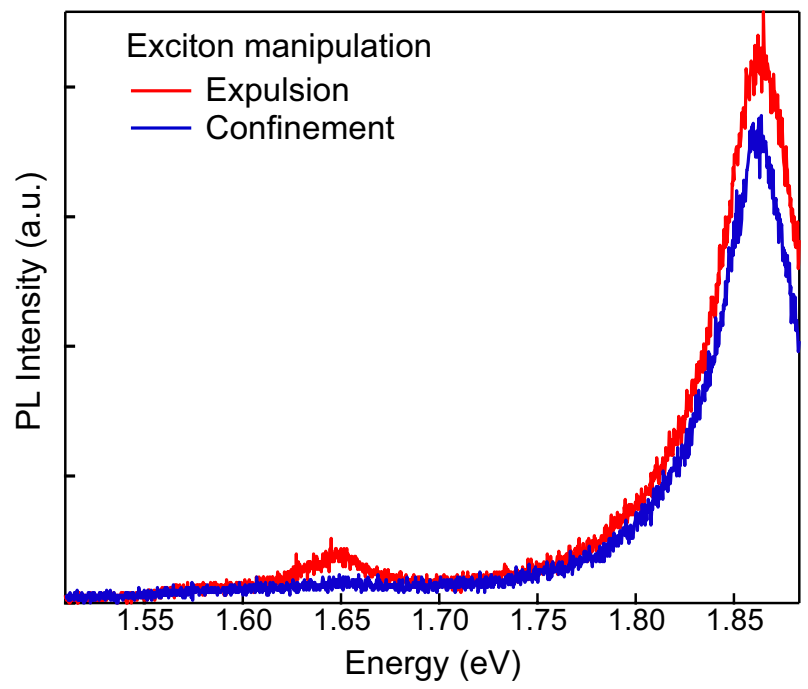

and red, respectively; b). c, d, Intensity distribution of light emission from the excitonic device in the confinement and expulsion configurations (left and right, respectively; c) and the corresponding spectra collected from the entire device (blue and red, respectively; $\mathbf{d}$ ). 
Single-particle transport hypothesis
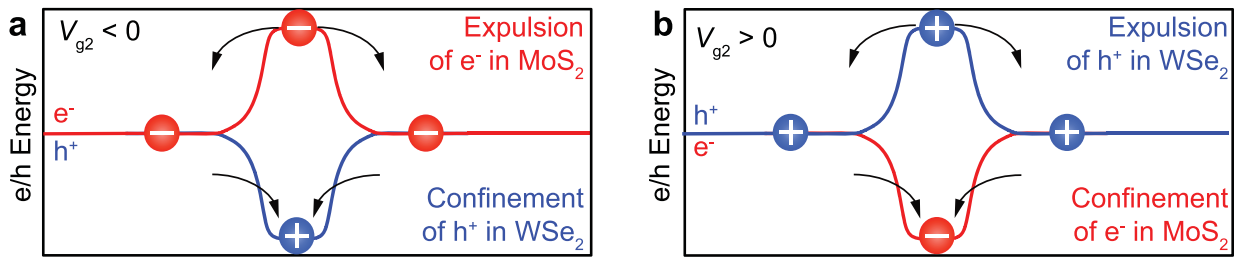

C

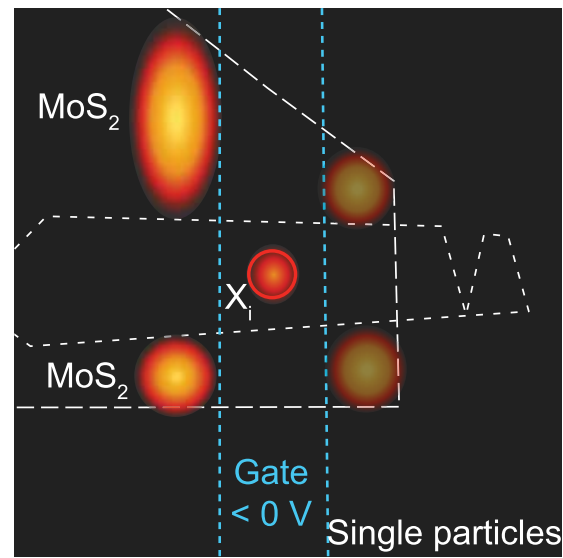

d

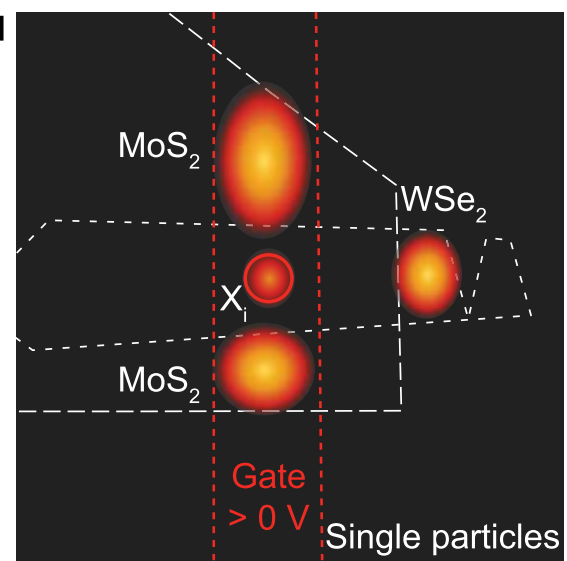

Experimental observations (ILE)

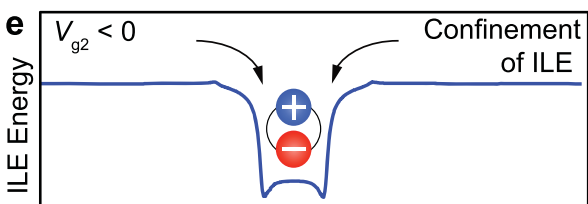

g

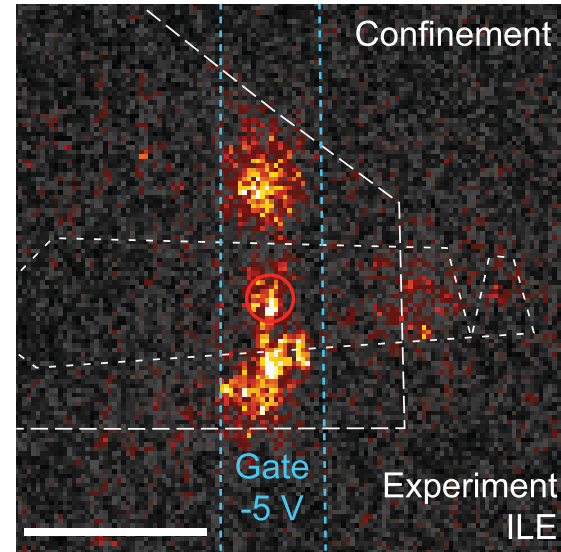

Extended Data Fig. 7 | Schematic depiction of the control over the light emission. a, b, Energy profiles for electrons (red) and holes (blue) in the device when applying negative (a) or positive (b) voltage on the central gate $\left(V_{\mathrm{g} 2}\right)$. c, d, Corresponding expected emission images under the singleparticle assumption. e, f, Energy profiles of interlayer excitons (ILE) in the presence of an external electric field, under the same conditions as in $\mathbf{a}$ and $\mathbf{b}$. g, h, Corresponding experimental results. Scale bar, $5 \mu \mathrm{m}$. The figures in $\mathbf{a}-\mathbf{d}$ are schematics based on the hypothesis that after the fast interlayer charge transfer, photo-excited carriers move independently rather than being bound in interlayer excitons. The diffusion of single electrons and holes is then subject to the type-II band alignment between $\mathrm{MoS}_{2}$ and $\mathrm{WSe}_{2}$, which restricts the motion of electrons to $\mathrm{MoS}_{2}$ and holes to $\mathrm{WSe}_{2}$. This charge separation is very efficient, as indicated by the strong suppression of intralayer emission from the heterostructure (Fig. 1e, f). Once the separation occurs, it is not very likely that the charges can hop between the layers: the band difference between $\mathrm{MoS}_{2}$ and WSe $\mathrm{Wi}_{2}$ more than $200 \mathrm{meV}$, so thermal excitation of $25 \mathrm{meV}$ will not be enough for electrons to jump back to $\mathrm{WSe}_{2}$ and holes to jump back to $\mathrm{MoS}_{2}$. Another thing to consider is the local electrostatic potential defined by

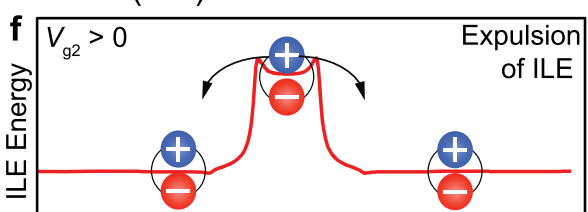

h

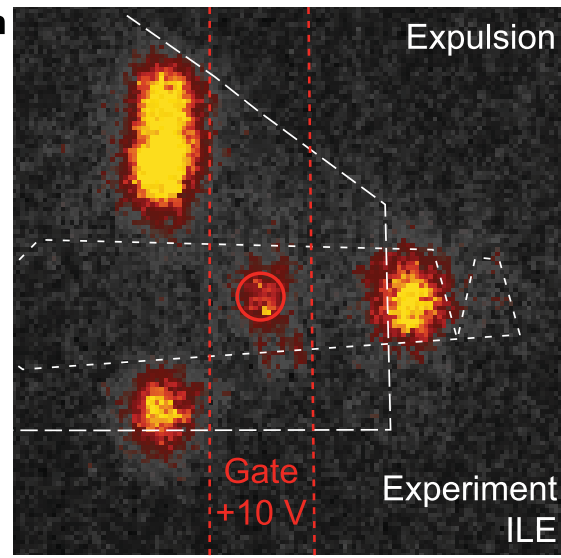

the gate. The application of $V_{\mathrm{g} 2}<0$ creates a confining energy profile for single holes and a repulsive one for single electrons, as in a and c. Holes would then be confined in the $\mathrm{WSe}_{2}$ area under the gate while electrons would be pushed out to $\mathrm{MoS}_{2}$ areas next to the gate, where they would recombine with charges already present in the monolayer area, resulting in photoluminescence from single-layer areas of $\mathrm{MoS}_{2}$ next to the gate (provided that there are enough holes in $\mathrm{MoS}_{2}$ to start with). We would then expect to obtain the emission pattern shown in c, assuming the presence of native holes in $\mathrm{MoS}_{2}$. In their absence, we would expect to see only one emission spot, coinciding with the excitation laser spot. Along the same lines, applying a positive gate voltage to the middle gate $\left(V_{\mathrm{g} 2}>0\right)$ would result in a repulsive potential for holes in $\mathrm{WSe}_{2}$ and an attractive one for electrons in $\mathrm{MoS}_{2}$. Recombination would then occur for electrons in $\mathrm{MoS}_{2}$ in regions under the gate and for holes in $\mathrm{WSe}_{2}$ in regions outside the gate, as shown in $\mathbf{d}$. This is in contradiction with the experimental observations in $\mathbf{e}-\mathbf{h}$. In the case of interlayer exciton transport, we instead have only a single energy profile $(\mathbf{e}, \mathbf{g})$, and the application of a positive voltage on the middle gate results in the expulsion of interlayer excitons from the injection region $(\mathbf{f}, \mathbf{h})$. 


\section{RESEARCH LETTER}

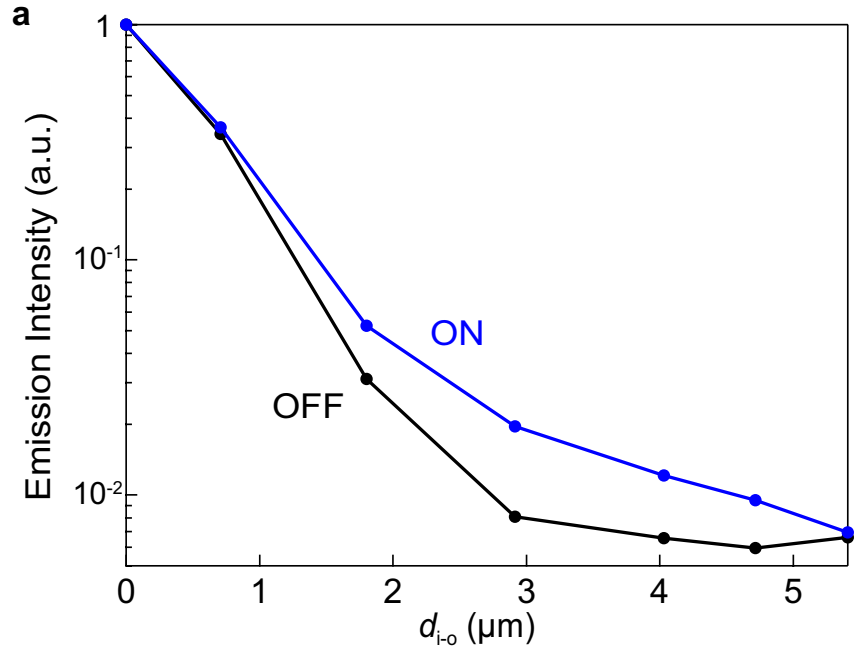

Extended Data Fig. 8 | Excitonic transistor characterization for different positions of the excitation laser spot. a, Normalized emission intensity (transistor output) as a function of the distance between optical injection and the emission point $d_{\mathrm{i}-\mathrm{o}}$, which is the same as in Fig. 3c, shown for the ON (blue, $V_{\mathrm{g} 1}=0 \mathrm{~V}$ ) and OFF (black, $V_{\mathrm{g} 1}=16 \mathrm{~V}$ ) states. b

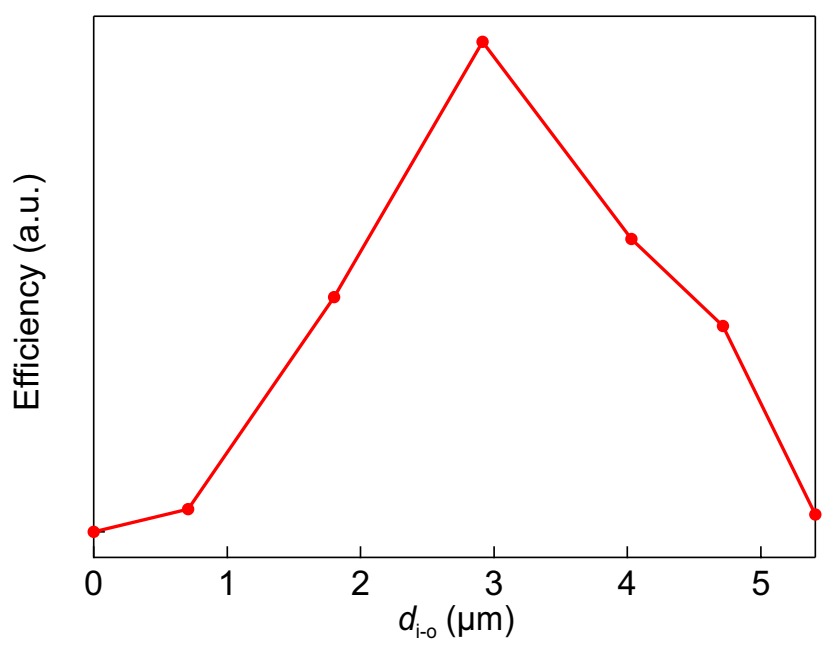

b, Transistor efficiency calculated as the ratio between output emission in the ON and OFF states for different input-output separation distances $d_{\mathrm{i}-\mathrm{o}}$. Efficiency reaches a maximum when the laser spot is moved completely beyond the gate, so that the energy barrier stays between the input and the output and thus effectively modulates exciton diffusion. 
a

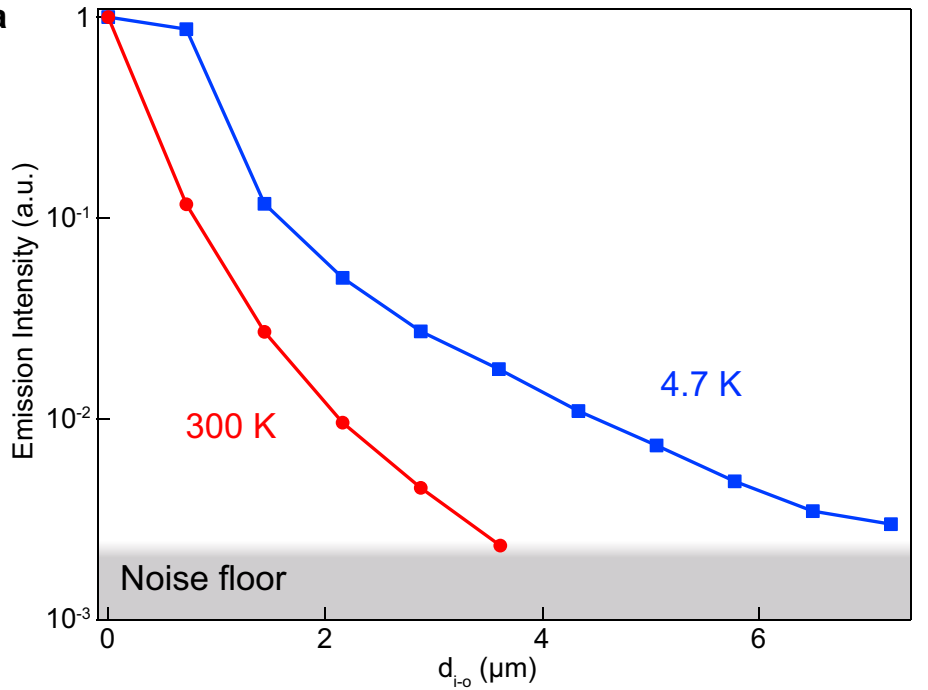

b
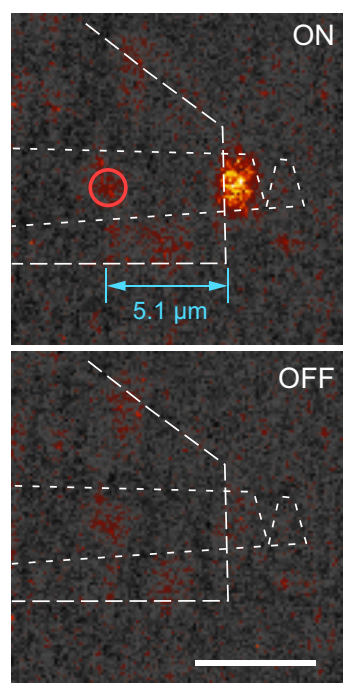

Extended Data Fig. 9 | Characterization of the device at low temperatures. a, Normalized output intensity as a function of the distance between optical injection and emission points, obtained at room temperature (red, $300 \mathrm{~K}$ ) and $4.7 \mathrm{~K}$ (blue). No electric field is applied. b, Emission images of the device in the ON (top) and OFF (down) states when measured at $4.7 \mathrm{~K}$, with input-output separations as long as $d_{\mathrm{i}-\mathrm{o}}=5.1 \mu \mathrm{m}$. Such long-distance transistor switching was not observed at room temperature for this sample. 


\section{RESEARCH LETTER}

\section{a}

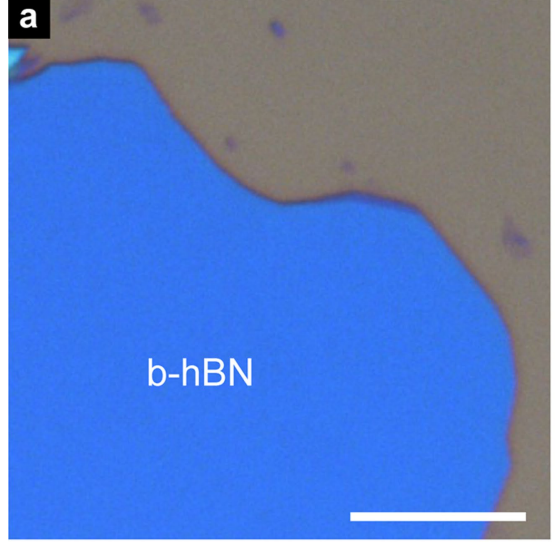

\section{d}

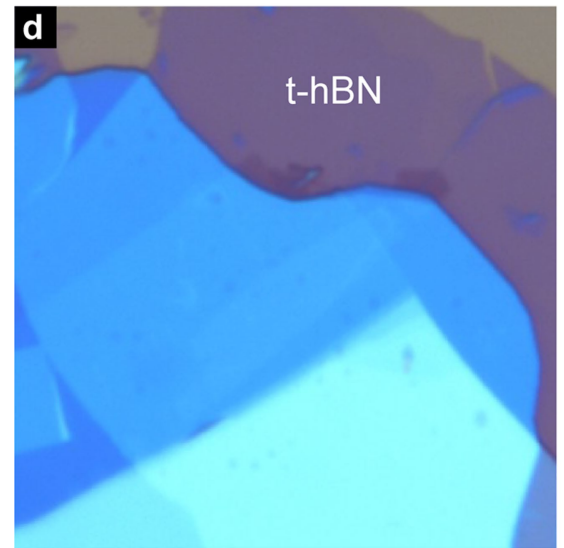

Extended Data Fig. 10 | Heterostructure fabrication. Optical images taken during different fabrication steps: a, exfoliation of the bottom h-BN (b-hBN); b, transfer of a monolayer $\mathrm{MoS}_{2}$ flake; $\mathbf{c}$, transfer of a monolayer $\mathrm{WSe}_{2}$ flake; d, encapsulation with top h-BN ( $\left.\mathrm{t}-\mathrm{hBN}\right)$; e, transfer of

e
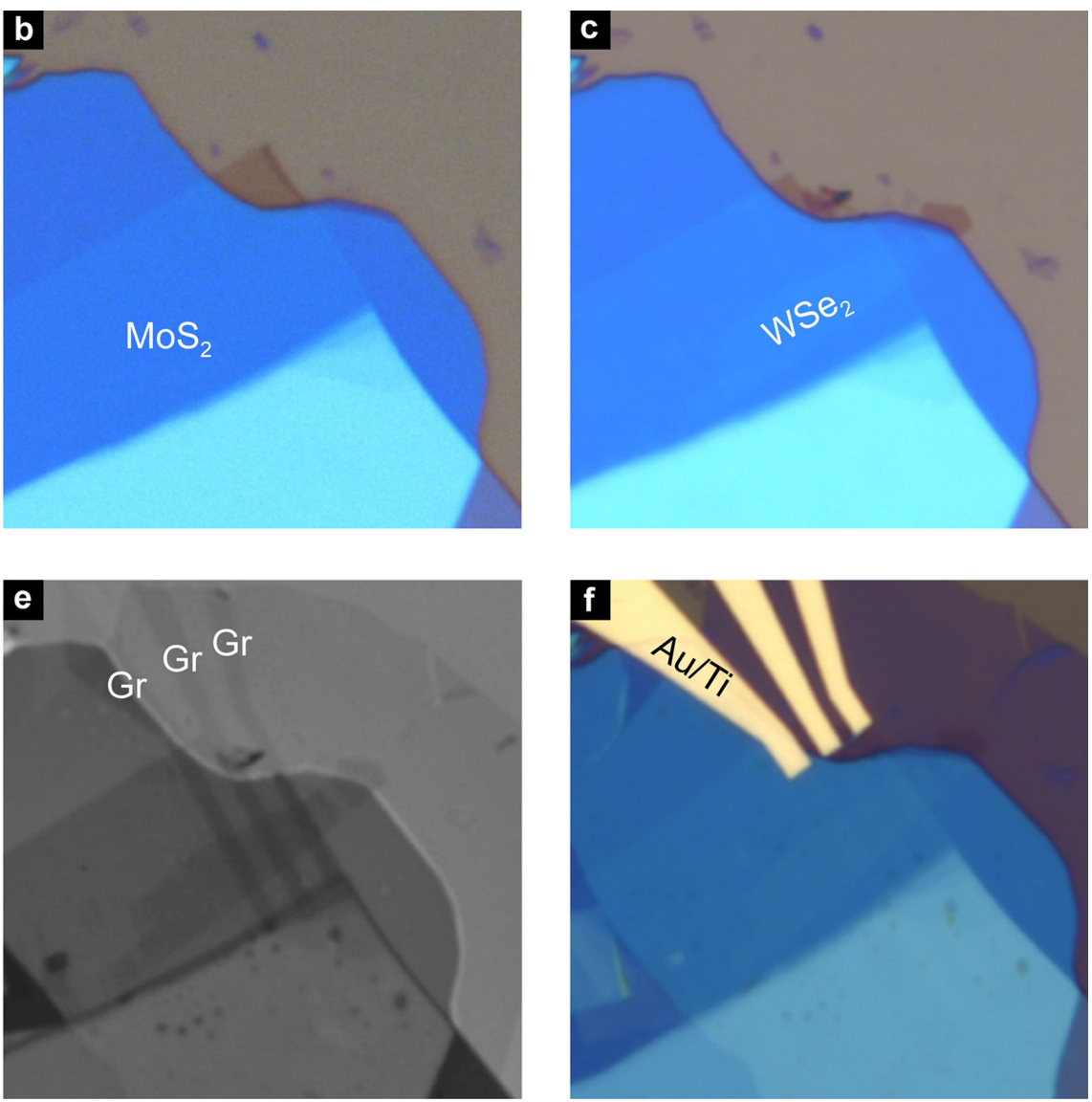

pre-patterned few-layer graphene stripes (Gr); and $\mathbf{f}$, metallization of $\mathrm{Au} / \mathrm{Ti}$ contacts. The image in $\mathbf{e}$ is shown in black and white for better visibility of the final structure. Scale bar, $10 \mu \mathrm{m}$ (applies to all images). 
a

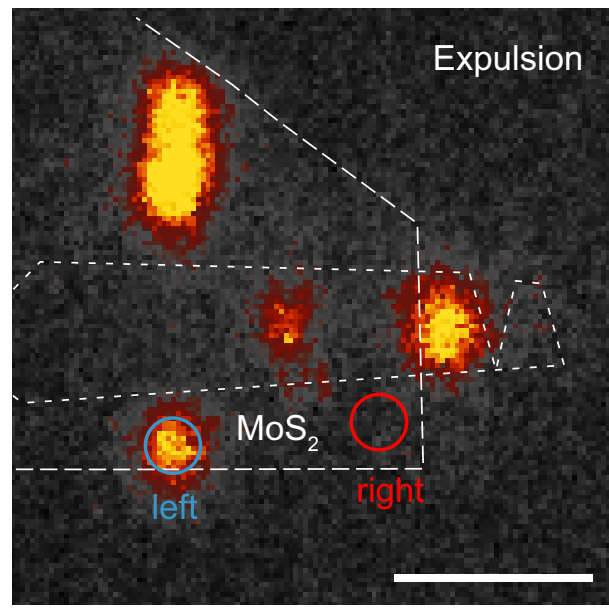

Extended Data Fig. 11 | Variation in photoluminescence emission from $\mathrm{MoS}_{2}$ due to the inhomogeneity of the substrate. a, Image of photoluminescence emission coming from the device in the repulsive configuration shown in Fig. 4f. b, Micro-photoluminescence $(\mu \mathrm{PL})$ spectra from the areas marked by red and blue circles in a, showing different peak

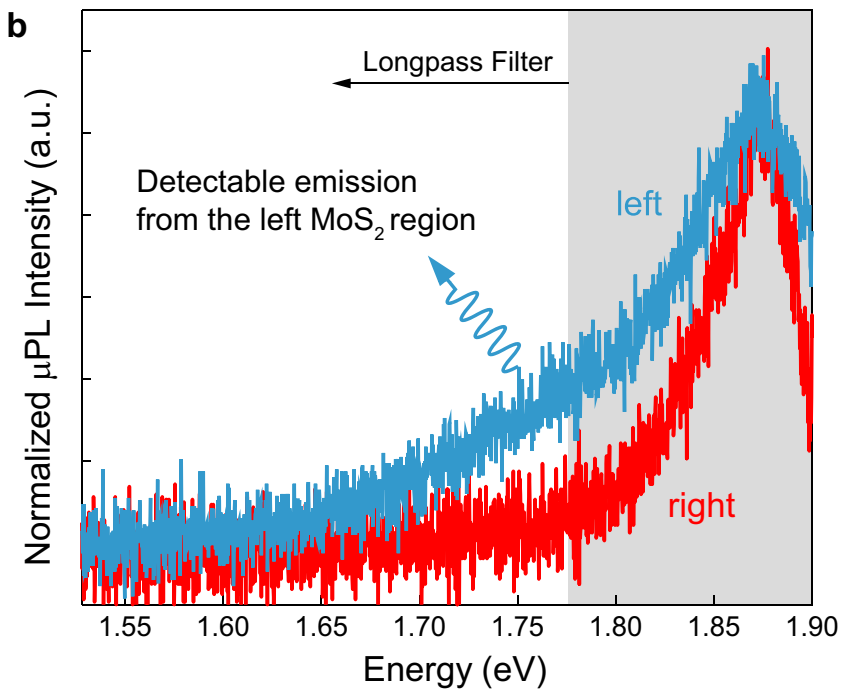

widths as a result of local inhomogeneity in the heterostructure. The grey shaded area is the part of the spectrum cut by the 700-nm long-pass filter. As can be clearly seen in the image, areas where $\mathrm{MoS}_{2}$ photoluminescence shows a low-energy tail due to broadening become visible to the CCD (left side of the device), whereas the other areas appear dark (right side). 
a

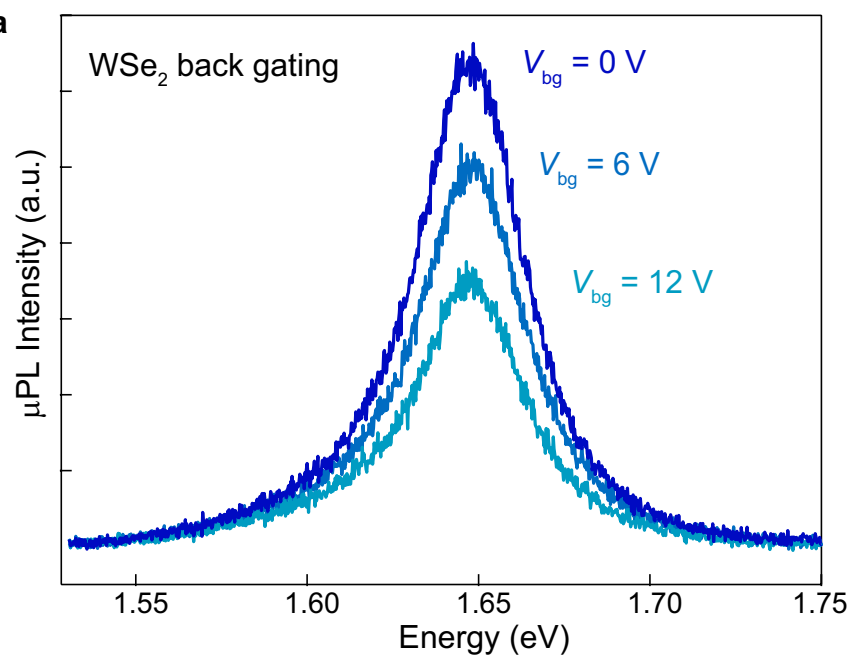

Extended Data Fig. $12 \mid$ Reference experiments. a, Photoluminescence spectra from monolayer $\mathrm{WSe}_{2}$ at different back-gate voltages. Substantial modulation of the emission intensity is observed. $\mathbf{b}$, Photoluminescence spectra from monolayer $\mathrm{WSe}_{2}$ when using top and back gates in the b

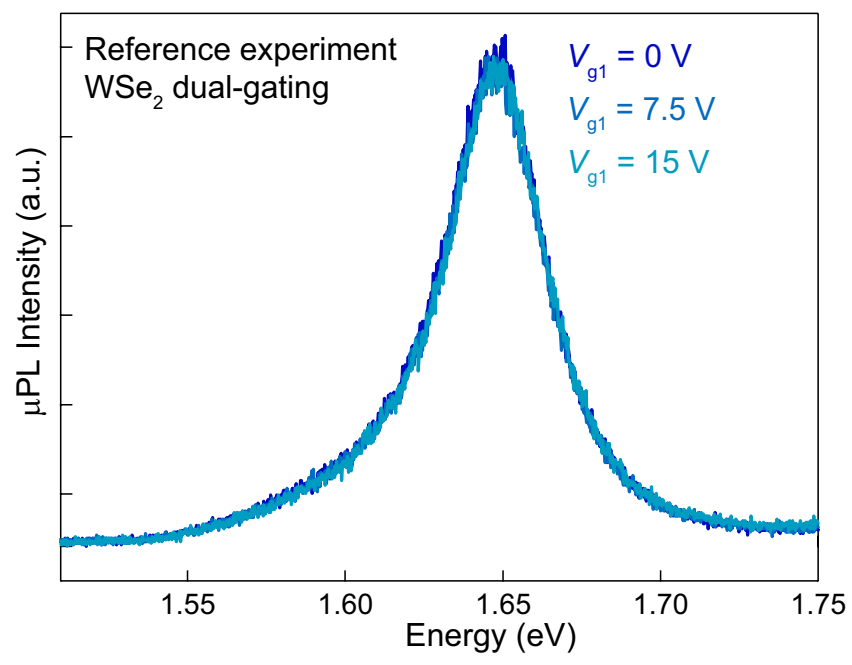

dual-gated configuration, for the voltage range used in the experiment presented in Fig. 2. No appreciable intensity modulation is observed. Both measurements are performed on the same $\mathrm{WSe}_{2}$ flake with the same continuous-wave excitation at $647 \mathrm{~nm}$ and $200 \mu \mathrm{W}$ of incident power. 


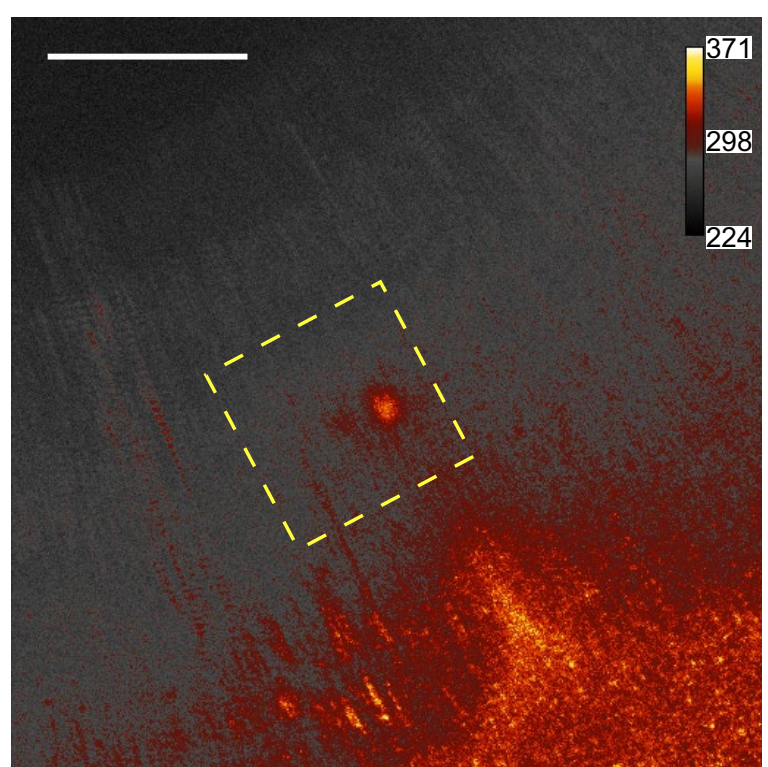

C

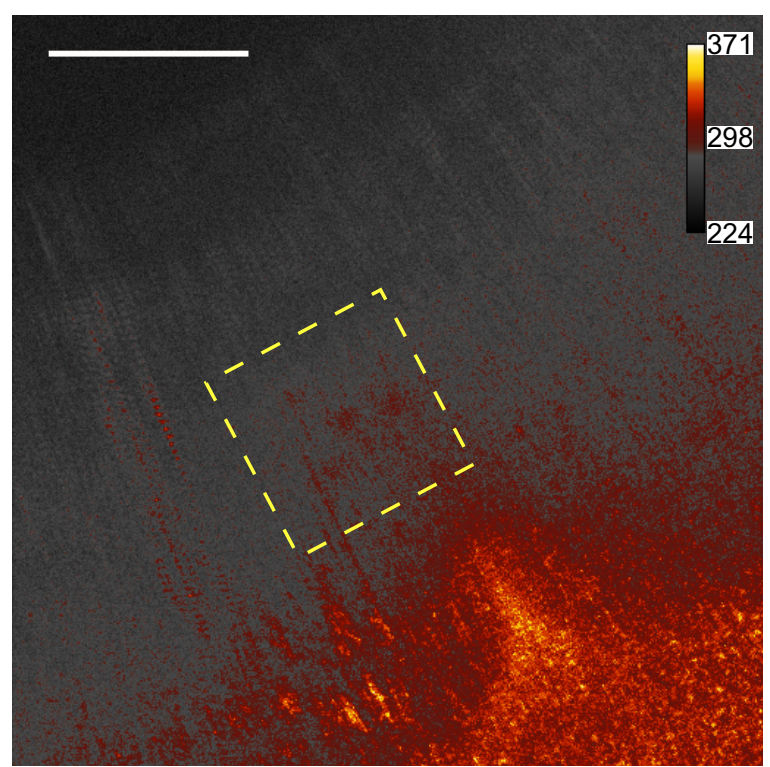

Extended Data Fig. 13 | Image post-processing. a, Original CCD image of the exciton emission for the configuration shown in Fig. 3a. The yellow square highlights the area of interest, shown in Fig. 3c. b. The same image b

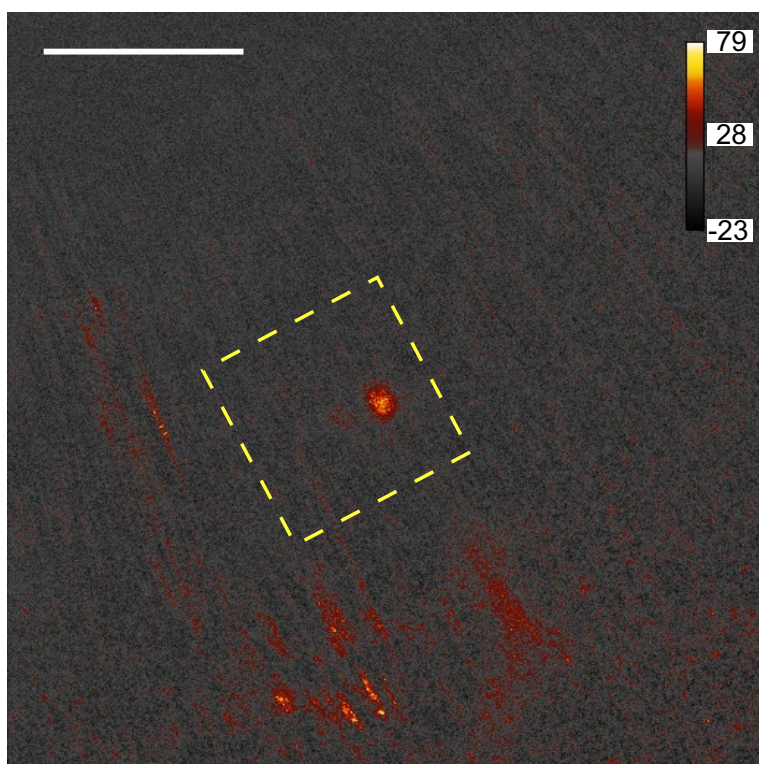

d

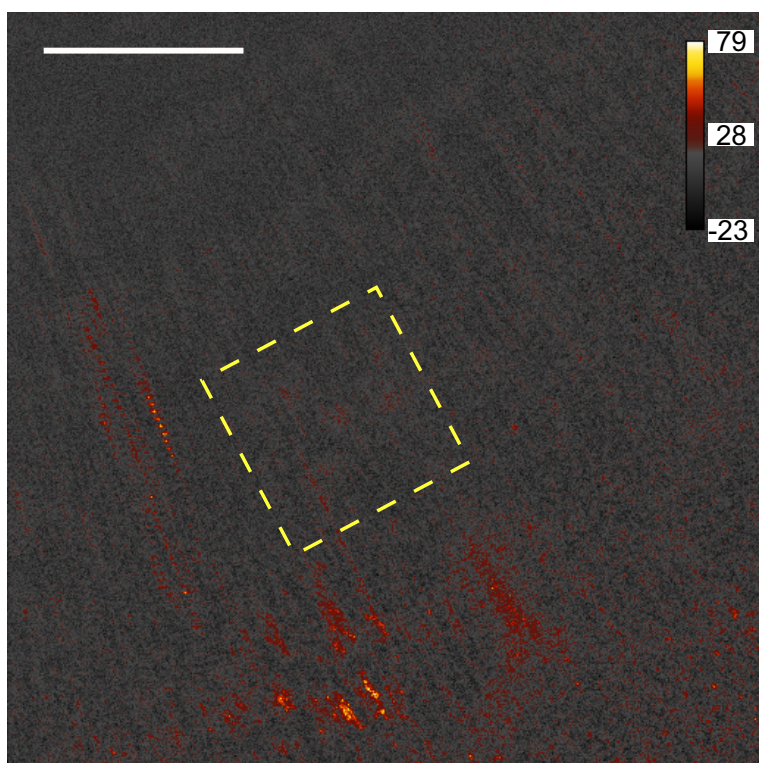

after background subtraction. $\boldsymbol{c}$, Original CCD image of the exciton emission for the configuration shown in Fig. 3b. d, The same image after background subtraction. Scale bars, $15 \mu \mathrm{m}$. 


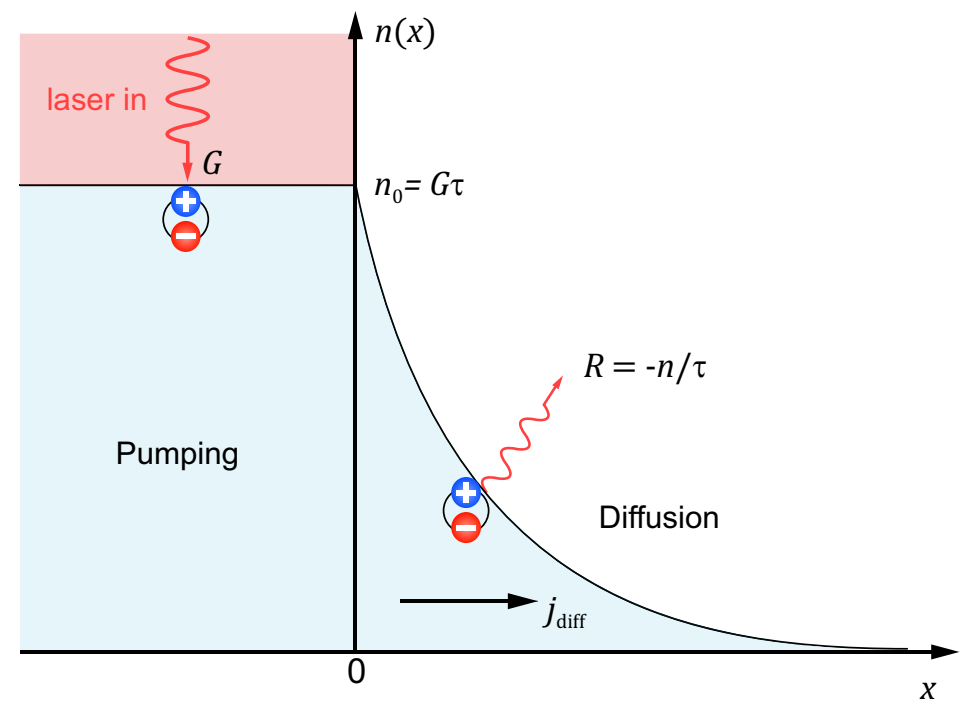

Extended Data Fig. $14 \mid$ Modelling of exciton diffusion. Schematic of exciton generation in the pumping area $(x<0)$ and diffusion outside $(x>0)$, represented by the exciton concentration $n(x)$. Constant pumping by the laser (red area) is determined by the generation rate $G$. This rate together with the recombination rate $R$ establish the exciton concentration $n_{0}$ in the pumping region. The concentration gradient outside the pumping area generates an exciton flux $j_{\text {diff }}$ that drives diffusion and leads to the exponential decay of exciton concentration along the $x$ axis. 
a

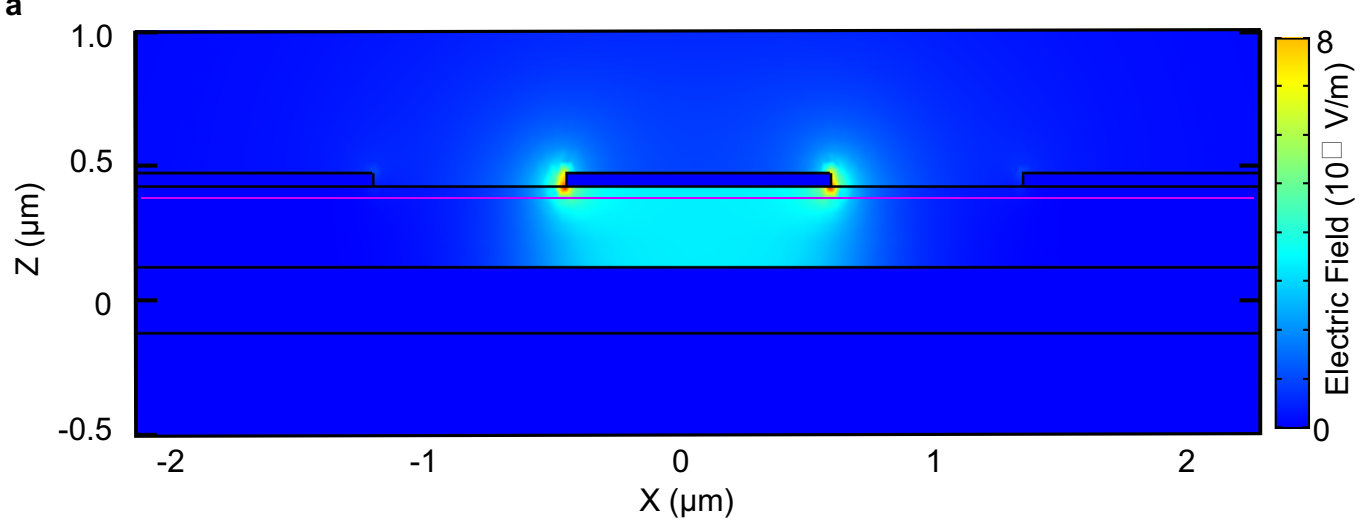

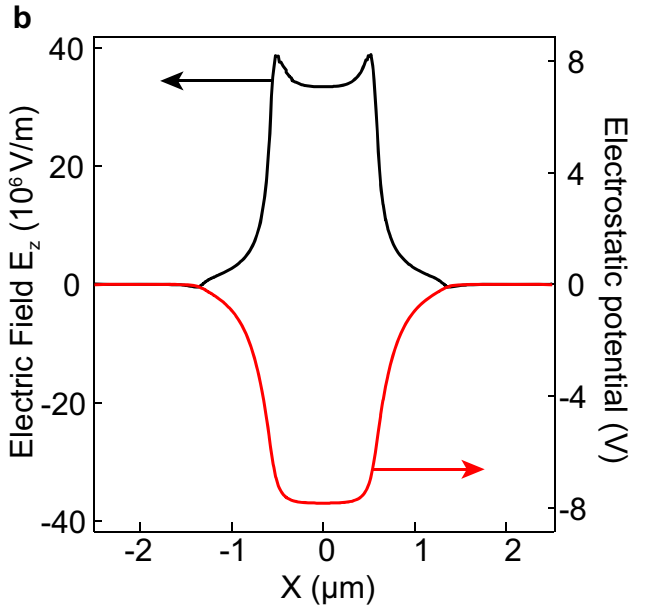

c

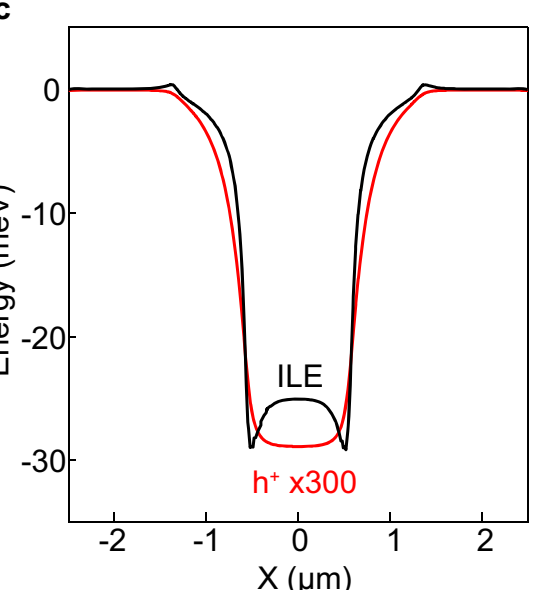

d

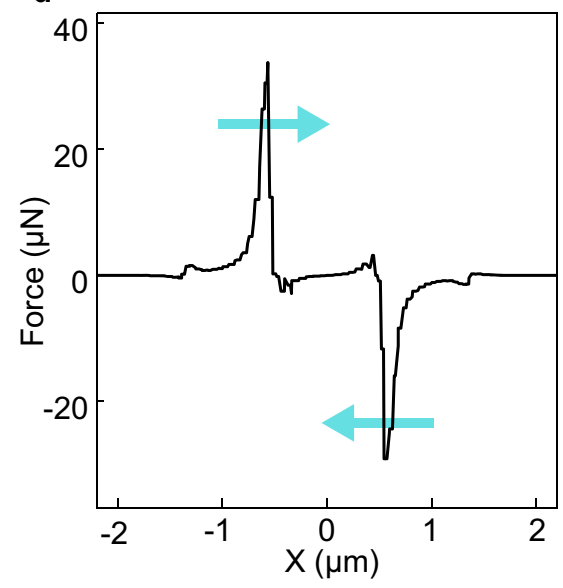

Extended Data Fig. 15 | Numerical simulation of the interlayer exciton in an external field. a, Two-dimensional cross-sectional map of the electric-field amplitude calculated for the device in the exciton confinement configuration, with $-10 \mathrm{~V}$ applied to the central gate and the side gates grounded. $\mathbf{b}$, Corresponding amplitude of the vertical electric field (black, left axis) and the electrostatic potential (red, right axis) along the heterobilayer. c, Energy shift experienced by an interlayer exciton (black, ILE) and a single hole (red, multiplied by 300 ) along the same cross-section. d, Projection along the $x$ axis of the confinement force experienced by the interlayer exciton owing to the presence of the electric field. Blue arrows show the direction of the force. 